\title{
Handbook for Surface Flow Velocity Measurement
}

\author{
Borbála Hortobágyi ${ }^{1}$, Matthew T. Perks ${ }^{1}$, Andrew J. Russell ${ }^{1}$, Alejandro Dussaillant ${ }^{2}$ \\ ${ }^{1}$ Newcastle University, School of Geography, Politics and Sociology, Daysh Building, \\ Claremont Road, Newcastle-upon-Tyne, NE1 7RU, UK \\ ${ }^{2}$ Universidad de Aysén, Campus Río Simpson, Patagonia-Aysén, Obispo Vielmo 62, \\ Coyhaique, Chile
}

Corresponding author: Borbála Hortobágyi (borbala.hortobagyi@newcastle.ac.uk)

\begin{abstract}
Acquisition of real-time hydraulic data is an essential component for flood forecasting. However, we frequently face difficulties in obtaining discharge data using classical contact methods during high magnitude floods and for systems experiencing rapid hydrogeomorphological adjustment. Therefore, we developed low-cost, non-contact sensors and platforms that are designed to overcome these difficulties. These advances enable flood flow properties to be monitored at multiple locations across a river catchment, at low-cost, and communicated in near real-time by using an image velocimetry method. This is an optics-based approach for stream flow measurement using commercially available nearinfrared digital cameras to acquire video footage in full HD (30fps). Video footage is then subjected to optical flow tracking techniques based on cross-correlation, and feature-based tracking, enabling the displacement rates of detected features (for example natural foam, seeds, woody debris, and turbulent structures) to be computed. This manual provides step by step guidance to install an image-based gauging station. It contains the list of necessary components, the calibration process of a new camera and the assembly procedure of the system.
\end{abstract}

Keywords: flow surface velocity; handbook; non-contact river monitoring; low-cost; realtime 


\section{Background}

River water is a vital resource and its supply and management is fundamental for societal well-being and economic development. Globally, floods represent $47 \%$ of all weatherrelated disasters and over recent decades have seen increases in occurrence with consequent increases in losses to life and infrastructure in both developing and developed countries (UNISDR, 2015). As such, there is a pressing need to improve our ability to reduce flood risk for less developed countries where sparse hydrometric monitoring networks and poor communications hamper the ability to forecast floods and to provide real-time flood alerts.

Risk to life, infrastructure and property is

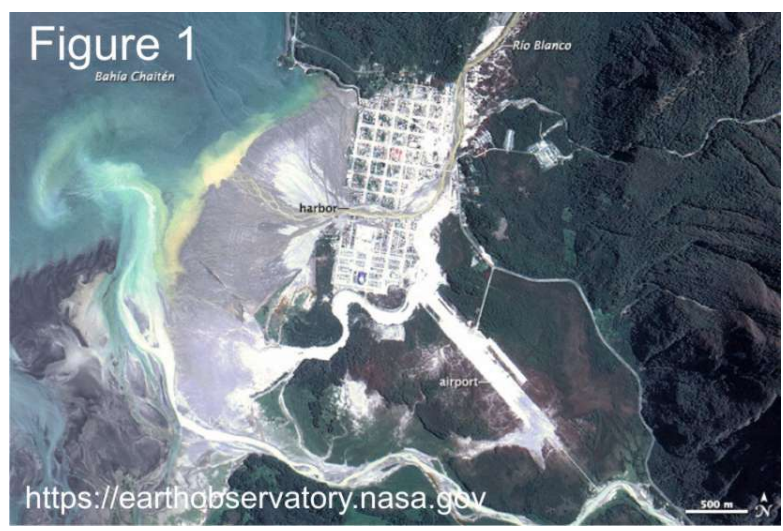
considerably enhanced when flood waters are charged with sediment and floating debris. Enhanced stream powers during high magnitude floods drive erosion and deposition of sediment which in turn bring about significant changes in channel cross-sectional geometry. Within-flood processes have been responsible for major river channel avulsions into populated areas (Major et al. 2016; Wilcox et al., 2016). Increased supply of sediment to fluvial systems through natural or anthropogenic processes can also have a major impact on downstream river channel dynamics resulting in localised aggradation and the downstream passage of sediment waves. Large volumes of woody debris may also be introduced suddenly to fluvial systems via landslides or following volcanic activity. Such hydrogeomorphological activity poses a major hazard to population and infrastructure in both developed and developing nations.

Acquisition of real-time hydraulic data is an essential component for flood forecasting, allowing validation of rainfall-runoff and hydraulic modelling approaches and providing information directly to hazard managers. However, the ability to obtain discharge data during high magnitude floods and for systems experiencing rapid hydro-geomorphological adjustment and is severely reduced by high sediment loads and floating debris which frequently clog conventional stage-recording equipment and compromise the integrity of stage-discharge relationships due to changes in channel cross-sectional geometry.

Difficulties involving the instrumentation of catchments deemed to be vulnerable to extreme runoff events in Chile, and other developing countries include: (i) the technical challenges of building accurate and resilient systems; (ii) the cost of implementation and maintenance, and (iii) access to adequately trained staff. Therefore, we developed low-cost non-contact sensors and platforms that are designed to overcome these difficulties. These advances enable flood flow properties to be monitored at multiple locations across a river catchment, at low-cost, and communicated in near real-time. This allows measurement of fundamental hydraulic processes, and provides the potential for enhanced flood warnings 
during high magnitude events when traditional river gauging methodologies often fail (Perks et al., 2016). This new data can be used for short term, early warning (to protect lives and infrastructure such as roads, bridges, drinking water plants, hydropower plants and dams), as well as longer-term areal coverage (where the established national network cannot cover due to costs or other considerations), with direct development implications including hazard maps and infrastructure site planning.

\section{Methodology}

Image velocimetry is an optics-based approach for stream flow measurement using commercially available near-infrared digital cameras to acquire video footage in full HD (30fps). Video footage is then subjected to optical flow tracking techniques based on crosscorrelation, and feature-based tracking, enabling the displacement rates of detected features (for example natural foam, seeds, woody debris, and turbulent structures) to be computed. First, we extract video frames from the footage, then, georeference them to convert image pixels to real-world coordinates. Second, we extract the start and end position of selected surface water features, then convert them to real-word coordinates to, finally, generate vectors of water velocities. The computation of water velocity vectors are achieved through application of several methodological approaches including large scale particle image velocimetry (LSPIV) (Muste et al. 2008, LeCoz et al. 2010), particle tracking velocimetry (PTV) (Tauro and Grimaldi 2017), and Kanade-Lucas-Tomasi (KLT) flow tracking (Perks et al. 2016). Following the determination of the surface velocity, a site-specific velocity coefficient can be calculated to translate surface velocities to depth-averaged velocities. To calibrate the relationship between surface velocity and discharge or water level, site-specific flow data (e.g. ADCP data) is necessary.

\section{Equipment}

The following table lists the major components necessary for an installation when a solar panel is used for power supply and an ultrasonic level sensor added. The use of an ultrasonic level sensor is optional; however additional hydraulic information (e.g. water surface slope) can be gained by installing a pair of downward facing ultrasonic water level sensors for mounting on bridges or structures where there is a vertical view angle. 


\begin{tabular}{|c|c|}
\hline Item & Example purchase outlet \\
\hline $\begin{array}{l}\text { Outdoor Cat5 } \\
\text { Ethernet cable } \\
(24 A W G)\end{array}$ & $\begin{array}{l}\text { https://www.amazon.com/Ubiquiti-Networks-TOUGHCable- } \\
\text { Shielded- } \\
\text { Ethernet/dp/B008L143VW/ref=cm cr arp d product top?ie=UTF8 }\end{array}$ \\
\hline $\begin{array}{l}\text { short Mini USB } \\
\text { cable for Pi to } 3 g \\
\text { module }\end{array}$ & $\begin{array}{l}\text { https://www.amazon.co.uk/System-S-Mini-USB-Cable-10- } \\
\text { cm/dp/B004A9JJ3G }\end{array}$ \\
\hline $45 \mathrm{~mm}$ hole saw & $\begin{array}{l}\text { https://www.amazon.co.uk/Diamond-Tipped-Ceramic-Glass- } \\
\text { Drill/dp/B00899CDZO }\end{array}$ \\
\hline $\begin{array}{l}\text { GEREE DC-DC } \\
\text { Converter Step } \\
\text { Down Module } \\
\text { 12V to 5V Micro } \\
\text { USB Output Buck } \\
\text { Power Adapter }\end{array}$ & $\begin{array}{l}\text { https://www.amazon.co.uk/dp/B01KX00QUU/ref=psdc } 340328031 \\
\text { t2 B00U2DGKOK }\end{array}$ \\
\hline $\begin{array}{l}\text { Solar panels } \\
100 \mathrm{~W} 12 \mathrm{~V}\end{array}$ & $\begin{array}{l}\text { http://cl.rsdelivers.com/product/rs-pro/stp100rsbp/rs-pro-100w- } \\
\text { monocrystalline-solar-panel/9046143 }\end{array}$ \\
\hline $\begin{array}{l}\text { Solar panel } \\
\text { ground mount }\end{array}$ & $\begin{array}{l}\text { https://uk.rs-online.com/web/p/solar-panel-mounting- } \\
\text { accessories/8602890/ }\end{array}$ \\
\hline $\begin{array}{l}\text { Solar controller } \\
(20 \mathrm{~A})\end{array}$ & https://uk.rs-online.com/web/p/products/9054536/ \\
\hline $\begin{array}{l}\text { High capacity } 12 v \\
\text { Deep cycle } \\
\text { batteries }\end{array}$ & $\begin{array}{l}\text { http://cl.rsdelivers.com/product/rs-pro/rs120-12/rs-pro-12v-lead- } \\
\text { acid-battery-120ah/7270401 }\end{array}$ \\
\hline 12v cable (thick) & $\begin{array}{l}\text { http://cl.rsdelivers.com/product/rs-pro/1-3182y25-bk-100r/rs-pro-2- } \\
\text { core-25-mm-power-cable-black-polyvinyl/8114362 }\end{array}$ \\
\hline $12 \mathrm{v}$ cable (thin) & $\begin{array}{l}\text { http://cl.rsdelivers.com/product/nexans/h03vvh2f-2x075mm2-noir- } \\
\text { 5/nexans-2-core-075-mm-mains-power-cable-black/4390802 }\end{array}$ \\
\hline $\begin{array}{l}\text { Jump } \\
\text { Imale }\end{array}$ & 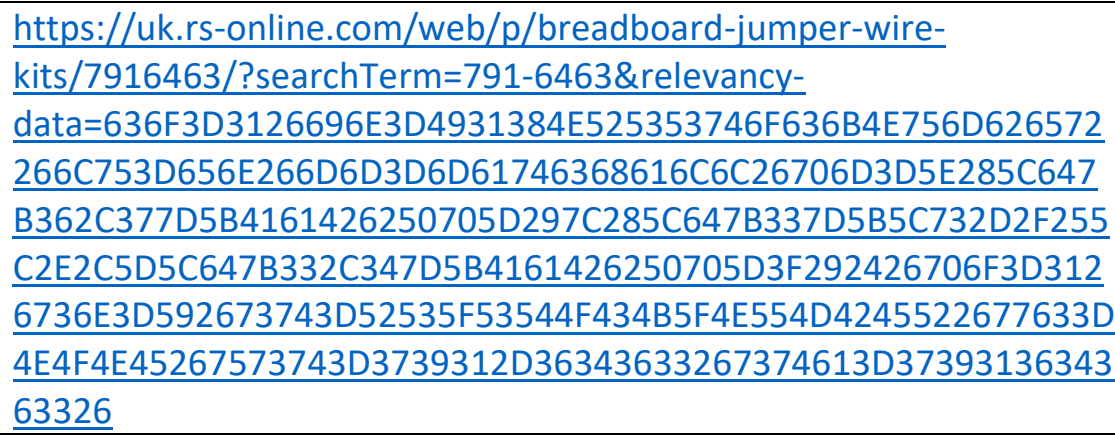 \\
\hline $\begin{array}{l}\text { Jumper cables } \\
\text { (female to male) }\end{array}$ & $\begin{array}{l}\text { https://uk.rs-online.com/web/p/breadboard-jumper-wire- } \\
\text { kits/7916454/?searchTerm=791-6454\&relevancy- } \\
\text { data=636F3D3126696E3D4931384E525353746F636B4E756D626572 } \\
\text { 266C753D656E266D6D3D6D61746368616C6C26706D3D5E285C647 } \\
\text { B362C377D5B4161426250705D297C285C647B337D5B5C732D2F255 } \\
\text { C2E2C5D5C647B332C347D5B4161426250705D3F292426706F3D312 } \\
\text { 6736E3D592673743D52535F53544F434B5F4E554D4245522677633D } \\
\text { 4E4F4E45267573743D3739312D36343534267374613D37393136343 }\end{array}$ \\
\hline
\end{tabular}




\begin{tabular}{|c|c|}
\hline & $\underline{53426}$ \\
\hline $\begin{array}{l}\text { Jumper cables } \\
\text { (female to female) }\end{array}$ & $\begin{array}{l}\text { https://uk.rs-online.com/web/p/breadboard-jumper-wire- } \\
\text { kits/7916450/?searchTerm=791-6450\&relevancy- } \\
\text { data=636F3D3126696E3D4931384E525353746F636B4E756D626572 } \\
\text { 266C753D656E266D6D3D6D61746368616C6C26706D3D5E285C647 } \\
\text { B362C377D5B4161426250705D297C285C647B337D5B5C732D2F255 } \\
\text { C2E2C5D5C647B332C347D5B4161426250705D3F292426706F3D312 } \\
\text { 6736E3D592673743D52535F53544F434B5F4E554D4245522677633D } \\
\underline{\text { 4E4F4E45267573743D3739312D36343530267374613D37393136343 }}\end{array}$ \\
\hline $\begin{array}{l}\text { Raspberry Pi } \\
\text { controller model } \\
\text { 3B }\end{array}$ & $\begin{array}{l}\text { https://uk.rs-online.com/web/p/processor-microcontroller- } \\
\text { development-kits/8968660/ }\end{array}$ \\
\hline $\begin{array}{l}\text { Waterproof box } \\
\text { (internal) - } \\
\text { Polypropylene } \\
\text { IP55 Junction Box, } \\
150 \times 80 x \\
175 \mathrm{~mm} \text {, Grey }\end{array}$ & $\begin{array}{l}\text { http://cl.rsdelivers.com/product/schneider- } \\
\text { electric/enn05010/polypropylene-ip55-junction-box-150-x-80-x- } \\
\underline{175 \mathrm{~mm} / 0151808}\end{array}$ \\
\hline $\begin{array}{l}\text { Sensor cabling (RS } \\
\text { Pro } 6 \text { Core } \\
\text { Industrial Cable } \\
0.5 \mathrm{~mm}^{2} \mathrm{CSA}, \\
\text { Screened Black } \\
\text { Polyvinyl Chloride } \\
\text { PVC Sheath, 100m } \\
\text { Reel) }\end{array}$ & $\begin{array}{l}\text { http://cl.rsdelivers.com/product/rs-pro/rs-135/rs-pro-6-core- } \\
\text { industrial-cable-05-mm-csa-screened/6600495 }\end{array}$ \\
\hline $\begin{array}{l}\text { Maplin CAT } 6 \\
\text { Grey Coloured } \\
\text { Boots for RJ45 } \\
\text { Connectors } 10 \\
\text { Pack }\end{array}$ & $\begin{array}{l}\text { https://www.maplin.co.uk/p/maplin-cat-6-grey-coloured-boots-for- } \\
\text { ri45-connectors-10-pack-n60ee }\end{array}$ \\
\hline $\begin{array}{l}\text { MH Connectors } \\
\text { RJ45 RJ Connector } \\
\text { Boot, Grey }\end{array}$ & https://uk.rs-online.com/web/p/rj-connector-hoods-boots/1354155/ \\
\hline $\begin{array}{l}\text { Velcro Black Hook } \\
\& \text { Loop Tape, } \\
50 \mathrm{~mm} \times 2.5 \mathrm{~m}\end{array}$ & https://uk.rs-online.com/web/p/hook-loop-tapes/9181240/ \\
\hline $\begin{array}{l}\text { Nichicon } \\
\text { Aluminium } \\
\text { Electrolytic } \\
\text { Capacitor } 100 \mu \mathrm{F} \\
25 \mathrm{~V} \text { dc } 6.3 \mathrm{~mm} \\
\text { Through Hole PS } \\
\text { Series }+105^{\circ} \mathrm{C}\end{array}$ & $\begin{array}{l}\text { https://uk.rs-online.com/web/p/aluminium- } \\
\text { capacitors/5194059/?searchTerm=519-4059\&relevancy- } \\
\text { data=636F3D3126696E3D4931384E525353746F636B4E756D626572 } \\
\text { 266C753D656E266D6D3D6D61746368616C6C26706D3D5E285C647 } \\
\text { B362C377D5B4161426250705D297C285C647B337D5B5C732D2F255 } \\
\text { C2E2C5D5C647B332C347D5B4161426250705D3F292426706F3D312 } \\
\text { 6736E3D592673743D52535F53544F434B5F4E554D4245522677633D } \\
\underline{\text { 4E4F4E45267573743D3531392D34303539267374613D35313934303 }}\end{array}$ \\
\hline
\end{tabular}




\begin{tabular}{|c|c|}
\hline $\begin{array}{l}\text { Vishay CPF Series } \\
\text { E14 Axial Metal } \\
\text { Film Fixed } \\
\text { Resistor } 1 \mathrm{k} \Omega \pm 1 \% \\
2 \mathrm{~W} \pm 100 \mathrm{ppm} /{ }^{\circ} \mathrm{C}\end{array}$ & $\begin{array}{l}\text { https://uk.rs-online.com/web/p/through-hole-fixed- } \\
\text { resistors/8306848/?sra=pstk }\end{array}$ \\
\hline $\begin{array}{l}\text { Vishay CPF Series } \\
\text { Axial Metal Film } \\
\text { Fixed Resistor } \\
2.2 \mathrm{k} \Omega \pm 1 \% 2 \mathrm{~W} \\
\pm 100 \mathrm{ppm} /{ }^{\circ} \mathrm{C}\end{array}$ & 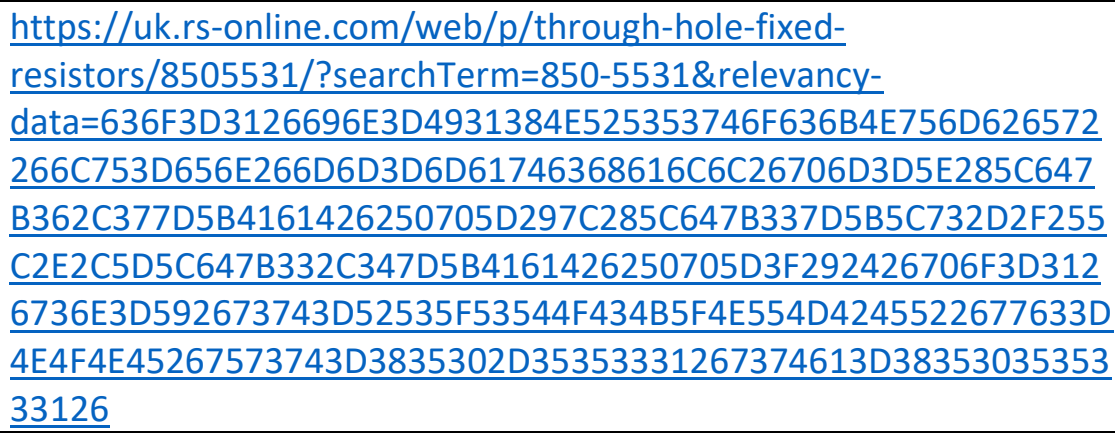 \\
\hline $\begin{array}{l}\text { Feed through } \\
\text { terminal block }\end{array}$ & $\begin{array}{l}\text { https://uk.rs-online.com/web/p/non-fused-din-rail- } \\
\text { terminals/0425207/?sra=pstk }\end{array}$ \\
\hline $\begin{array}{l}\text { Weidmuller } \\
1050000000, \text { End } \\
\text { Plate, W Series }\end{array}$ & $\begin{array}{l}\text { https://uk.rs-online.com/web/p/din-rail-terminal- } \\
\text { accessories/0425291/?origin=PSF 435779\%7Cacc }\end{array}$ \\
\hline $\begin{array}{l}\text { RS Pro Bi-metal } 16 \\
\rightarrow 51 \mathrm{~mm} 9 \text { piece } \\
\text { Hole Saw Set }\end{array}$ & https://uk.rs-online.com/web/p/hole-saw-sets/0216016/ \\
\hline $\begin{array}{l}\text { Acc Silicones } \\
740010370 \\
\text { Translucent } \\
\text { Silicone Potting } \\
\text { Compound } \\
\end{array}$ & $\begin{array}{l}\text { http://cl.rsdelivers.com/product/acc-silicones/740010370/acc- } \\
\text { silicones-740010370-translucent-silicone/4480242 }\end{array}$ \\
\hline $\begin{array}{l}\text { Black ABS Potting } \\
\text { Box with Lid, } 54 \times \\
38 \times 23 \mathrm{~mm}\end{array}$ & 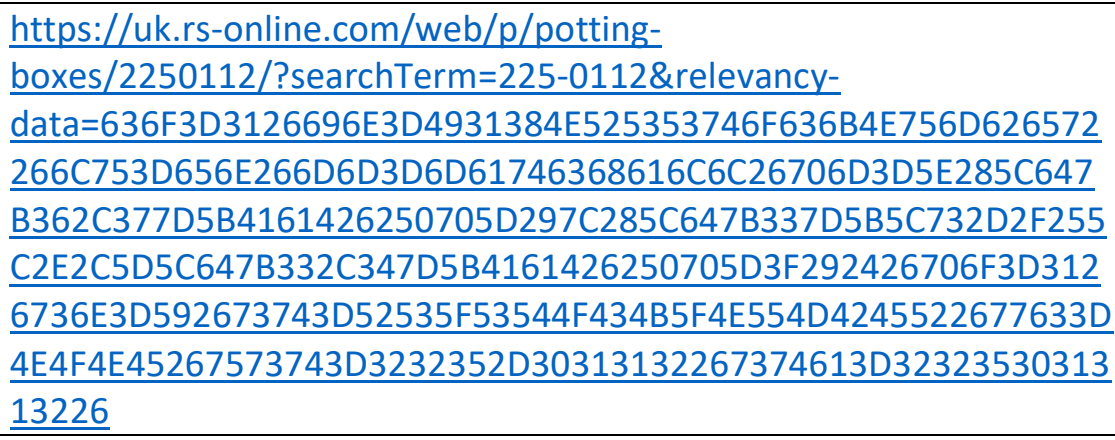 \\
\hline $\begin{array}{l}\text { Din Rail Top Hat, } \\
137 \mathrm{~mm} \times 35 \mathrm{~mm} \times \\
7.5 \mathrm{~mm}\end{array}$ & https://uk.rs-online.com/web/p/din-rails/2835690/ \\
\hline $\begin{array}{l}\text { Lapp M25 Grey } \\
\text { Polyamide, IP69K } \\
\text { Cable Gland With } \\
\text { Locknut }\end{array}$ & $\begin{array}{l}\text { https://uk.rs-online.com/web/p/cable- } \\
\text { glands/3658444/?origin=PSF } 412600 \% 7 \text { Cacc }\end{array}$ \\
\hline $\begin{array}{l}\text { Lapp M32 Grey } \\
\text { Polyamide, IP69K } \\
\text { Cable Gland }\end{array}$ & $\begin{array}{l}\text { https://uk.rs-online.com/web/p/cable- } \\
\text { glands/4442969/?origin=PSF 412600\%7Cacc }\end{array}$ \\
\hline Lapp Grey & https://uk.rs-online.com/web/p/cable-gland- \\
\hline
\end{tabular}




\begin{tabular}{|c|c|}
\hline $\begin{array}{l}\text { Fibreglass PA } \\
\text { Cable Gland } \\
\text { Locknut, M32 } \\
\text { Thread, IP68 }\end{array}$ & locknuts/4443013/?origin=PSF $412597 \% 7$ Cacc \\
\hline $\begin{array}{l}\text { Roline Cable } \\
\text { Crimper for RJ45, } \\
203 \mathrm{~mm}\end{array}$ & $\begin{array}{l}\text { http://cl.rsdelivers.com/product/roline/19061020-10/roline-cable- } \\
\text { crimper-for-rj45-203mm/1052153 }\end{array}$ \\
\hline Silica Gel, 10g & $\begin{array}{l}\text { https://uk.rs-online.com/web/p/humidity-indicating- } \\
\text { desiccators/0601041/?sra=pstk }\end{array}$ \\
\hline $\begin{array}{l}\text { RS Pro Crimp } \\
\text { terminal Kit, } \\
\text { Insulated } \\
\text { Terminal } \\
\end{array}$ & $\begin{array}{l}\text { http://cl.rsdelivers.com/product/rs-pro/yci-637/rs-pro-crimp- } \\
\text { terminal-kit-insulated-terminal/0534828 }\end{array}$ \\
\hline $\begin{array}{l}\text { RS Pro Insulated } \\
\text { Tin Plated Crimp } \\
\text { Ring Terminal, M6 } \\
\text { Stud Size, } 2.5 \mathrm{~mm}^{2} \\
\text { to } 6 \mathrm{~mm}^{2}, 12 \mathrm{AWG} \\
\text { to } 10 \mathrm{AWG}, \text { Yellow }\end{array}$ & https://uk.rs-online.com/web/p/products/0534430/ \\
\hline $\begin{array}{l}\text { RS Pro Insulated } \\
\text { Tin Plated Crimp } \\
\text { Ring Terminal, M5 } \\
\text { Stud Size, } 1.5 \mathrm{~mm}^{2} \\
\text { to } 2.5 \mathrm{~mm}^{2} \text {, } \\
\text { 16AWG to } \\
\text { 14AWG, Blue }\end{array}$ & https://uk.rs-online.com/web/p/products/0534575/ \\
\hline $\begin{array}{l}\text { RS Pro Insulated } \\
\text { Tin Plated Crimp } \\
\text { Ring Terminal, M5 } \\
\text { Stud Size, } 0.5 \mathrm{~mm}^{2} \\
\text { to } 1.5 \mathrm{~mm}^{2} \text {, } \\
\text { 22AWG to } \\
\text { 16AWG, Red }\end{array}$ & https://uk.rs-online.com/web/p/products/0534200/ \\
\hline $\begin{array}{l}\text { RS Pro DC, DC } \\
\text { Adapter Rated At } \\
1 \mathrm{~A}, 12 \mathrm{~V} \text {, Cable } \\
\text { Mount, length } \\
44 \mathrm{~mm} \text {, Nickel } \\
\text { Plate }\end{array}$ & https://uk.rs-online.com/web/p/dc-power-plugs/7719174/ \\
\hline $\begin{array}{l}\text { RS Pro DC, DC } \\
\text { Adapter Rated At } \\
1 \mathrm{~A}, 12 \mathrm{~V} \text {, Cable } \\
\text { Mount, length } \\
44 \mathrm{~mm} \text {, Nickel } \\
\text { Plate }\end{array}$ & https://uk.rs-online.com/web/p/dc-power-plugs/7719186/ \\
\hline $\begin{array}{l}\text { Conduit to protect } \\
\text { cables (Tower }\end{array}$ & $\begin{array}{l}\text { https://www.screwfix.com/p/tower-corrugated-conduit-black- } \\
\text { 20mm-x-10m/50443 }\end{array}$ \\
\hline
\end{tabular}




\begin{tabular}{|c|c|}
\hline $\begin{array}{l}\text { Corrugated } \\
\text { Conduit Black } \\
20 \mathrm{~mm} \times 10 \mathrm{~m} \text { ) } \\
\end{array}$ & \\
\hline $\begin{array}{l}\text { Waterproof box } \\
\text { (external) - IP55 } \\
\text { ENCLOSURE GREY } \\
270 \times 180 \times \\
135 \mathrm{MM}\end{array}$ & $\begin{array}{l}\text { https://www.screwfix.com/p/ip55-enclosure-grey-270-x-180-x- } \\
\text { 135mm/86625?kpid=86625\&gclid=Cj0KCQjwkZfLBRCzARIsAH3wMKr } \\
\text { WN0860bhOP1FUlkFAbBzA7nNvfg4LjNjCpN3boDS5pg0avpSibEsaAoN } \\
\text { XEALw wcB\&gclsrc=aw.ds\&dclid=CPvAml g9UCFUSC7QodeDkNkQ }\end{array}$ \\
\hline $\begin{array}{l}\text { RJ45 connection } \\
\text { tabs (Philex } \\
\text { PHILEX RJ45 8P8C } \\
\text { CONNECTORS } \\
\text { PACK OF 100) }\end{array}$ & $\begin{array}{l}\text { https://www.screwfix.com/p/philex-rj45-8p8c-connectors-pack-of- } \\
\underline{100 / 32033}\end{array}$ \\
\hline $\begin{array}{l}\text { JG SPEEDFIT } \\
\text { PEM0328WP } \\
\text { ELBOW WHITE } \\
28 \mathrm{MM}\end{array}$ & $\begin{array}{l}\text { https://www.screwfix.com/p/ig-speedfit-pem0328wp-elbow-white- } \\
\underline{28 \mathrm{~mm} / 86987}\end{array}$ \\
\hline $\begin{array}{l}\text { FLOPLAST PEX } \\
\text { PIPE } 28 M M \times 3 M\end{array}$ & https://www.screwfix.com/p/floplast-pex-pipe-28mm-x-3m/79799 \\
\hline $\begin{array}{l}\text { 3A TERMINAL } \\
\text { STRIPS PACK OF } \\
10\end{array}$ & $\begin{array}{l}\text { https://www.screwfix.com/p/3a-terminal-strips-pack-of- } \\
\text { 10/25545? requestid=194554\#product additional details container }\end{array}$ \\
\hline $\begin{array}{l}\text { Waterproof box } \\
\text { for battery } \\
\text { (REALLY USEFUL } \\
\text { BLACK 64L } \\
\text { PLASTIC STORAGE } \\
\text { BOX) }\end{array}$ & $\begin{array}{l}\text { http://www.sodimac.cl/sodimac-cl/product/3219925/Carro- } \\
\text { portaherramientas-36,5×64×40-cm-plastico/3219925 }\end{array}$ \\
\hline $\begin{array}{l}\text { Ultrasonic level } \\
\text { sensor }(5 \mathrm{~m}) \text { with } \\
\text { 3/4" NPS WR } \\
\text { Housing, Shielded } \\
\text { cable attach } \\
\text { option }\end{array}$ & https://www.maxbotix.com/Ultrasonic Sensors/MB7569.htm \\
\hline \multirow[t]{2}{*}{ OR } & https://www.maxbotix.com/Ultrasonic Sensors/MB7950.htm \\
\hline & https://www.maxbotix.com/Ultrasonic Sensors/MB7955.htm \\
\hline \multirow[t]{3}{*}{$\begin{array}{l}\text { Ultrasonic level } \\
\text { sensor }(10 \mathrm{~m}) \text { with } \\
3 / 4 " \text { NPS WR } \\
\text { Housing, Shielded } \\
\text { cable attach } \\
\text { option }\end{array}$} & https://www.maxbotix.com/Ultrasonic Sensors/MB7586.htm \\
\hline & https://www.maxbotix.com/Ultrasonic Sensors/MB7950.htm \\
\hline & https://www.maxbotix.com/Ultrasonic Sensors/MB7955.htm \\
\hline $\begin{array}{l}\text { 4G-3G-GPRS-GSM } \\
\text { External Antenna }\end{array}$ & https://www.cooking-hacks.com/4g-3g-gprs-gsm-antenna-external \\
\hline
\end{tabular}




\begin{tabular}{|c|c|}
\hline $\begin{array}{l}\text { PoE IR cams (short } \\
\text { range) - 4MP } \\
\text { Hikvision Hiwatch } \\
\text { IPC-B140 6MM } \\
55^{\circ} \text { Mini Bullet IP } \\
\text { Camera 30m EXIR }\end{array}$ & $\begin{array}{l}\text { https://www.hikvision.com/europe/Products/HiLook/Network- } \\
\text { Camera/4MP/IPC-B140 }\end{array}$ \\
\hline \multicolumn{2}{|l|}{ OR } \\
\hline $\begin{array}{l}\text { Hikvision DS- } \\
\text { 2CD2T42WD-I8 } \\
\text { 4MP EXIR } \\
\text { Network Bullet } \\
\text { Camera }\end{array}$ & $\begin{array}{l}\text { https://www.hikvision.com/en/Products/Network-Camera/EasylP- } \\
\text { 2.0/4MP/DS-2CD2T42WD-13/15/18 }\end{array}$ \\
\hline $\begin{array}{l}\text { Raspberry Pi to } \\
\text { Arduino Shields } \\
\text { Connection Bridge }\end{array}$ & $\begin{array}{l}\text { https://www.cooking- } \\
\text { hacks.com/documentation/tutorials/raspberry-pi-to-arduino-shields- } \\
\text { connection-bridge/ }\end{array}$ \\
\hline $\begin{array}{l}\text { 3G/GPRS shield } \\
\text { for Raspberry Pi } \\
\text { USA version }\end{array}$ & $\begin{array}{l}\text { https://www.cooking-hacks.com/3g-gprs-shield-for-raspberry-pi-3g- } \\
\text { gps }\end{array}$ \\
\hline $\begin{array}{l}\text { Maplin } 2.1 \times 5 \mathrm{~mm} \\
\text { DC Power Line } \\
\text { Socket }\end{array}$ & https://www.rapidonline.com/Catalogue/Product/20-1066 \\
\hline
\end{tabular}

Potentially needed:

Pipe clips for wall attachment:

https://www.screwfix.com/p/talon-28mm-hinged-pipe-clip-white-50-pack/58742

https://www.screwfix.com/p/km-28mm-snap-lid-single-hinged-clips-50-pack/30087 


\section{Camera calibration}

1. Connect the new camera to a PC via Ethernet and power using 12v DC.

2. Install (if necessary from the supplied CD) and load SADP software

3. List of connected devices is shown. If the camera hasn't been used previously it should show status as 'Inactive'.

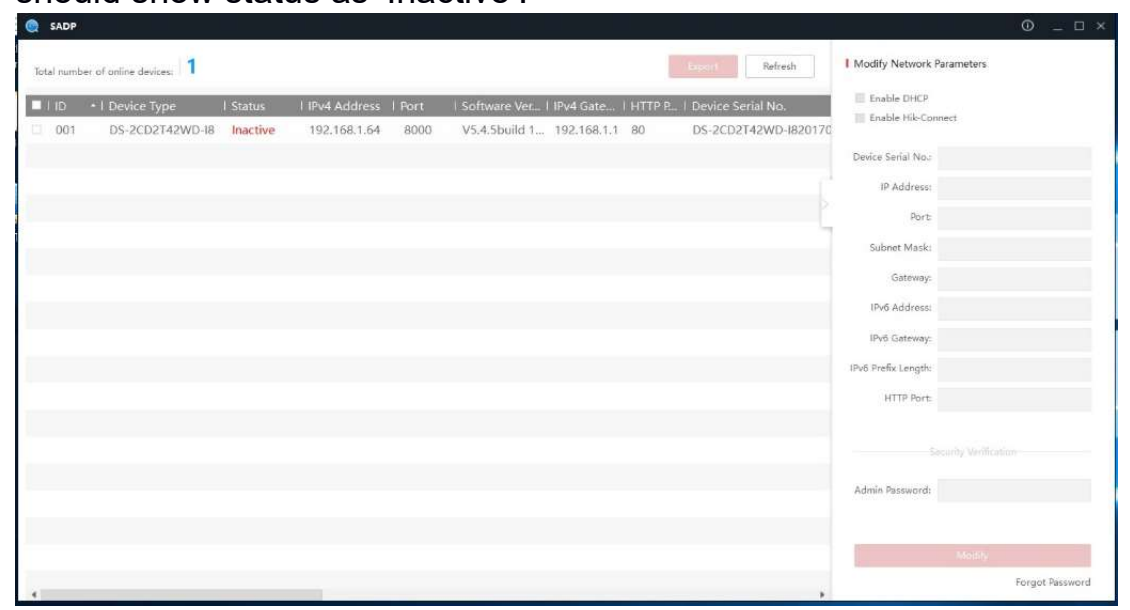

4. Click the checkbox next to the camera to display new popup window

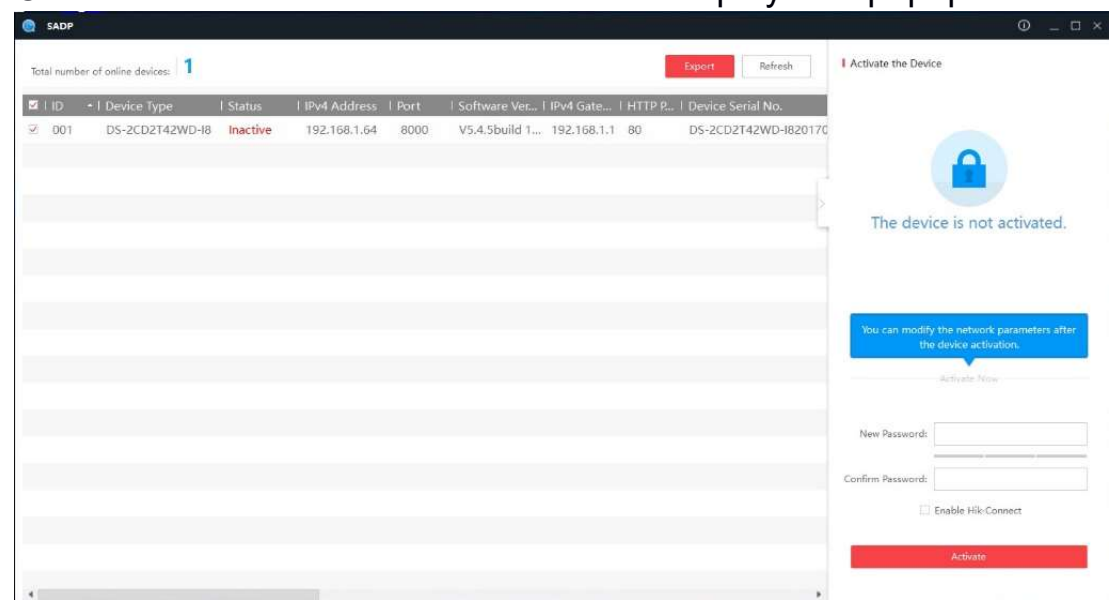

5. Type the password and confirm it. We use the password 'password11' (without apostrophes). Click activate. A popup saying device is activated should appear briefly.

6. Ensure that the IP address, subnet mask and Gateway are as default (192.168.1.64; 255.255.255.0; 192.168.1.1) and modify using the password (password11). Ensure Enable DHCP box is checked before exiting also 


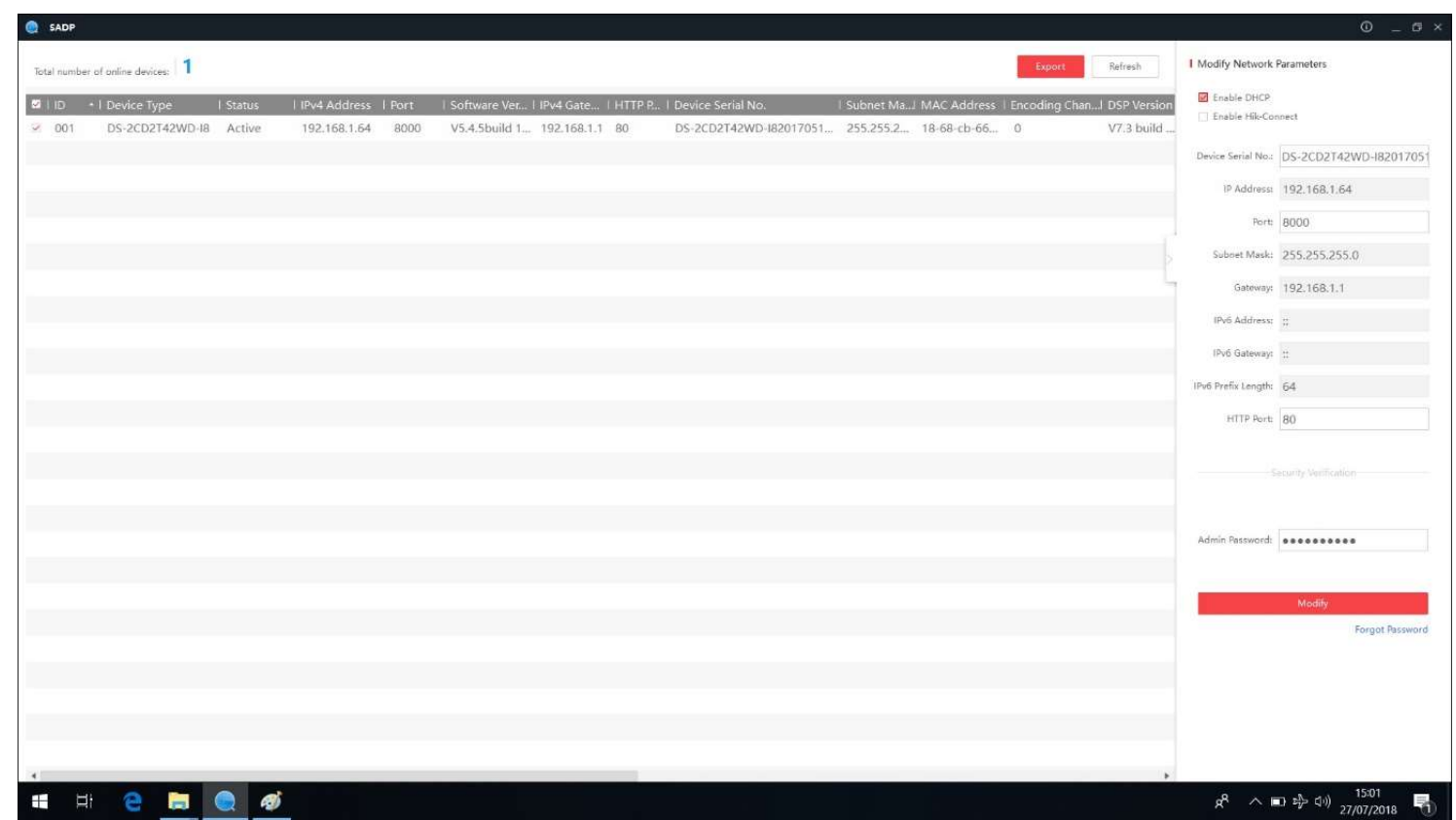

7. On your PC/laptop which is connected to the camera, disable all Wi-Fi connections and disconnect from the internet.

8. Go to Control Panel --- Click Network and Internet --- Network and sharing centre.

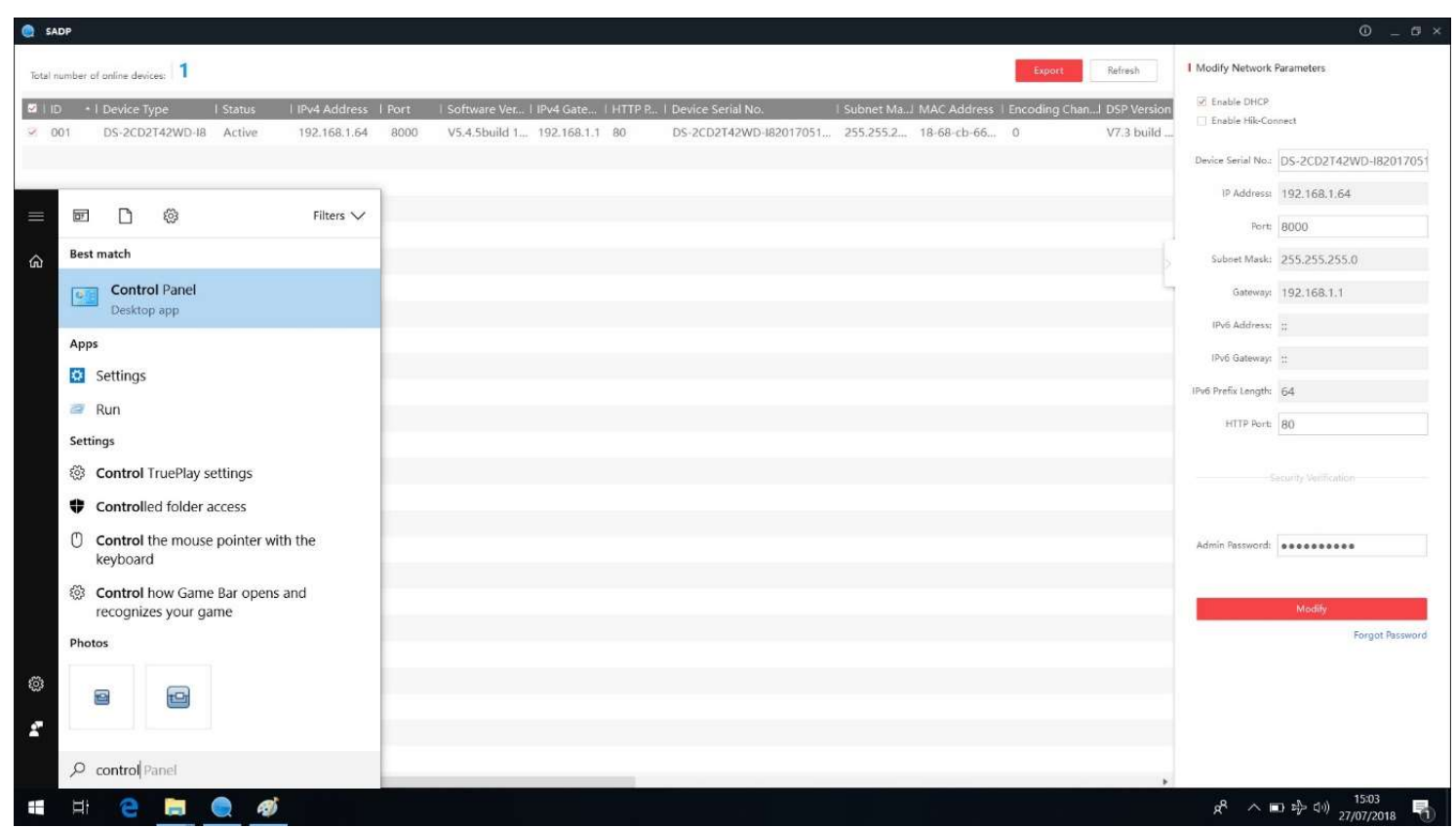



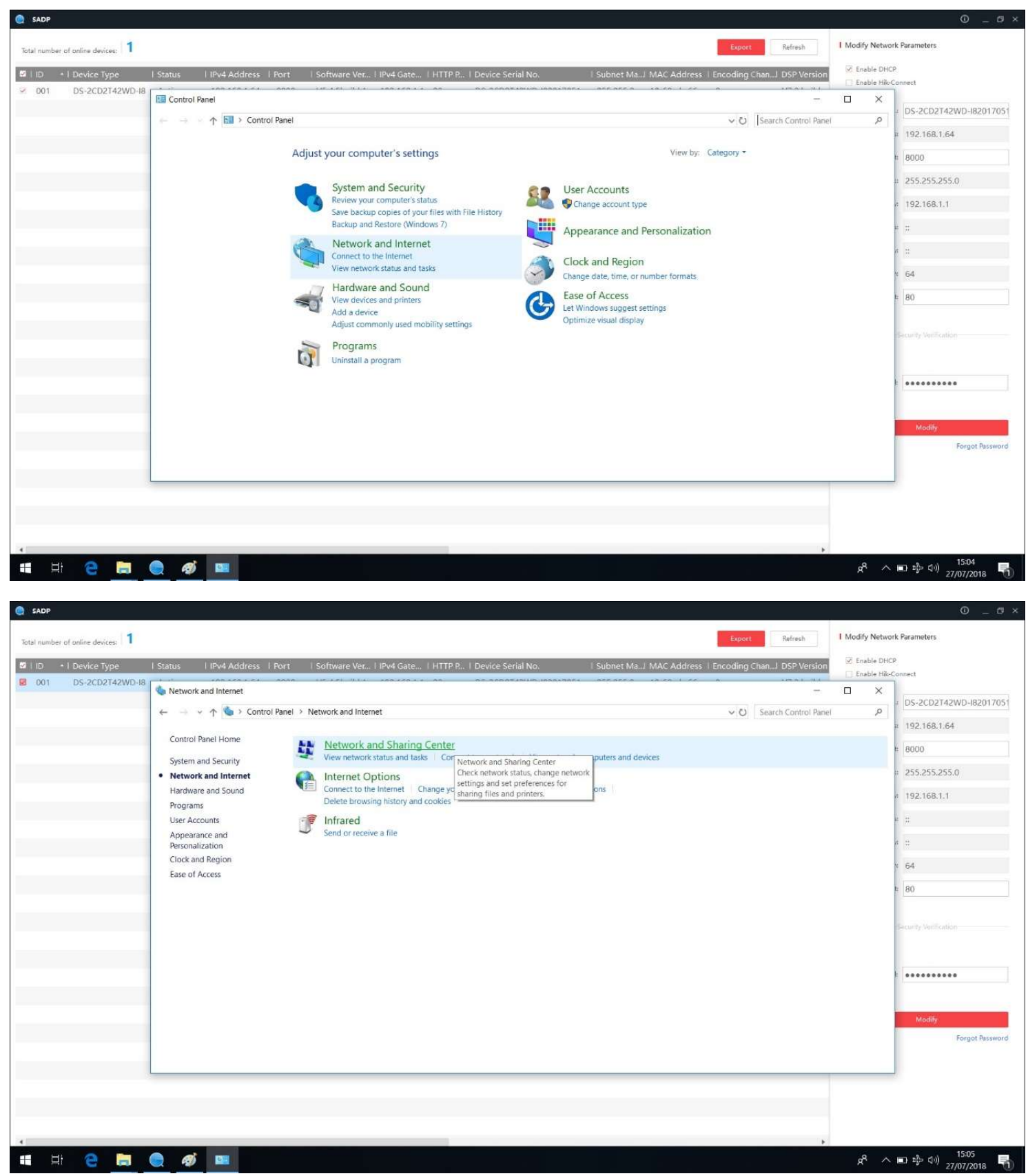

9. Click Change Adapter Settings (left hand side of the screen)

10. Right click on the active connection (this should be an Ethernet type). Click properties to open new window. Click on the writing 'Internet Protocol Version 4 (TCP/IPv4) and click properties. 


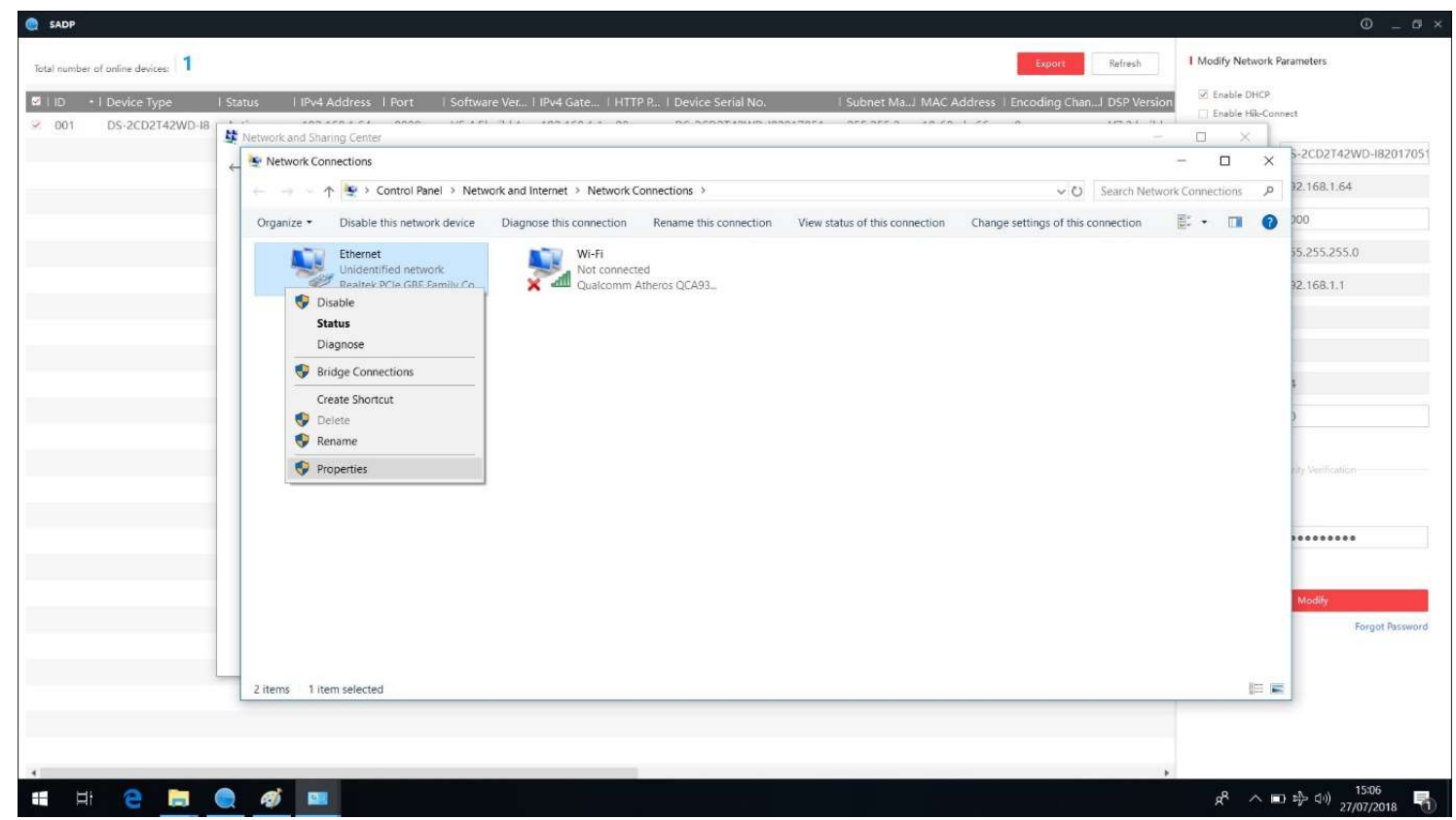

11. Change the general details (IP address, subnet mask and gateway) of the IP4 settings to as follows (192.168.1.100; 255.255.255.0; 192.168.1.1).

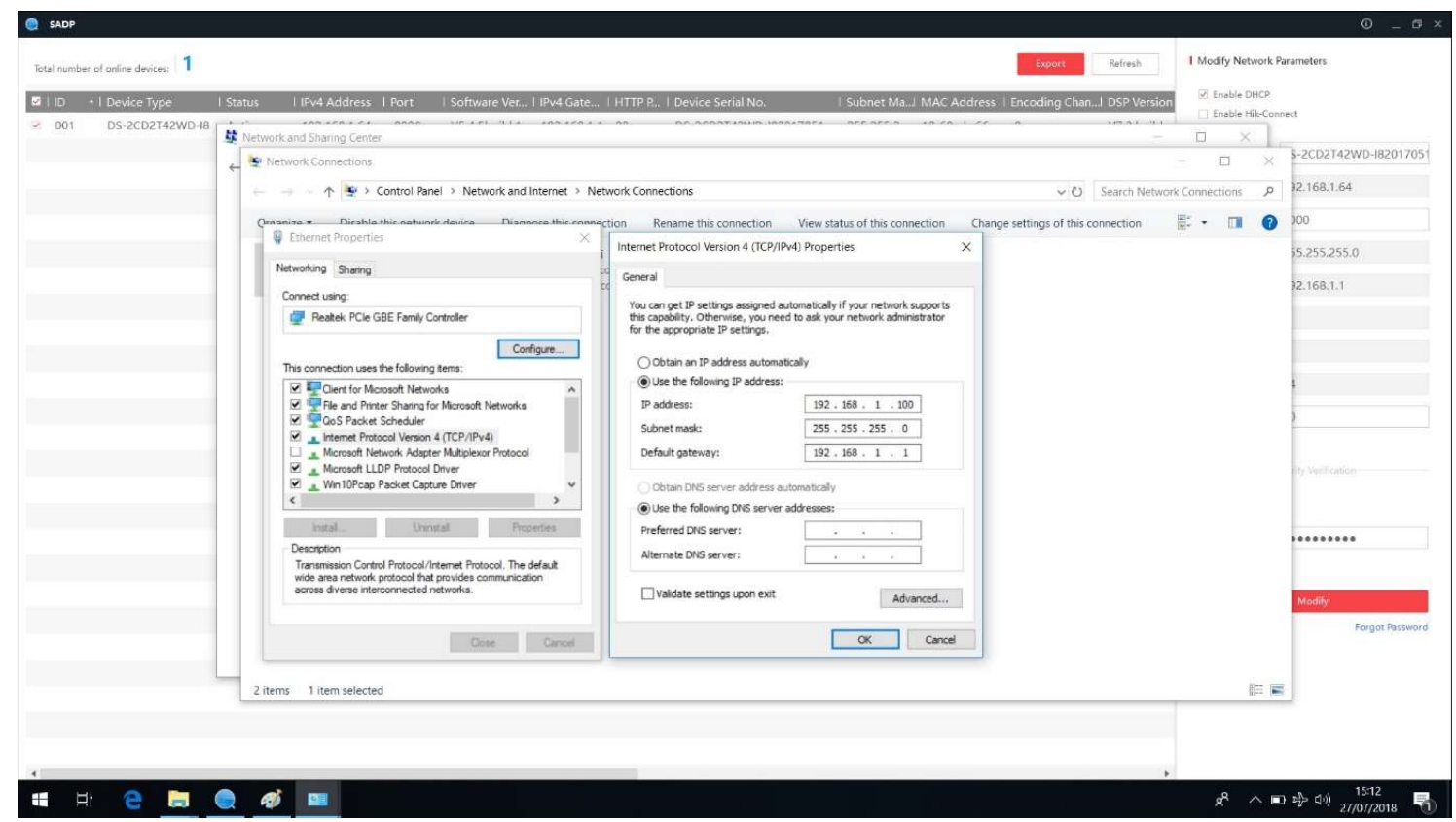

12. The camera can then be connected to via Internet Explorer or Chrome by using the address: 192.168.1.64. Settings can then be changed using this interface.

13. For the username type 'admin' (no quotes). For password type 'password11' (no quotes). Click login button. You are now logged into the camera and can change settings. 


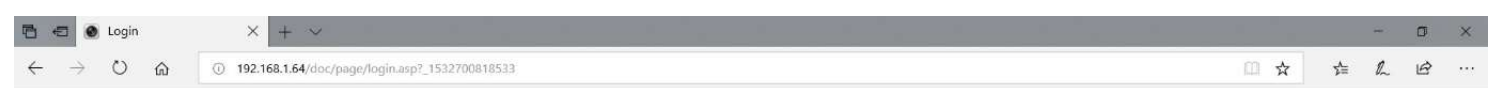

HIKVISION

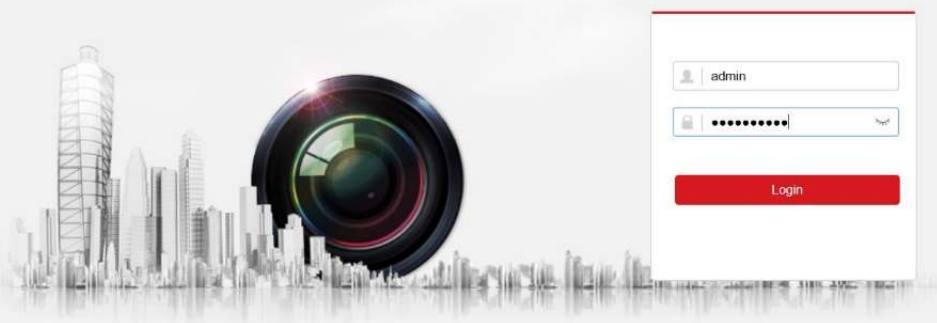

14. Click Configuration - Go to time settings --- Set the camera time manually to GMT $+00: 00)$ time. Click Save.

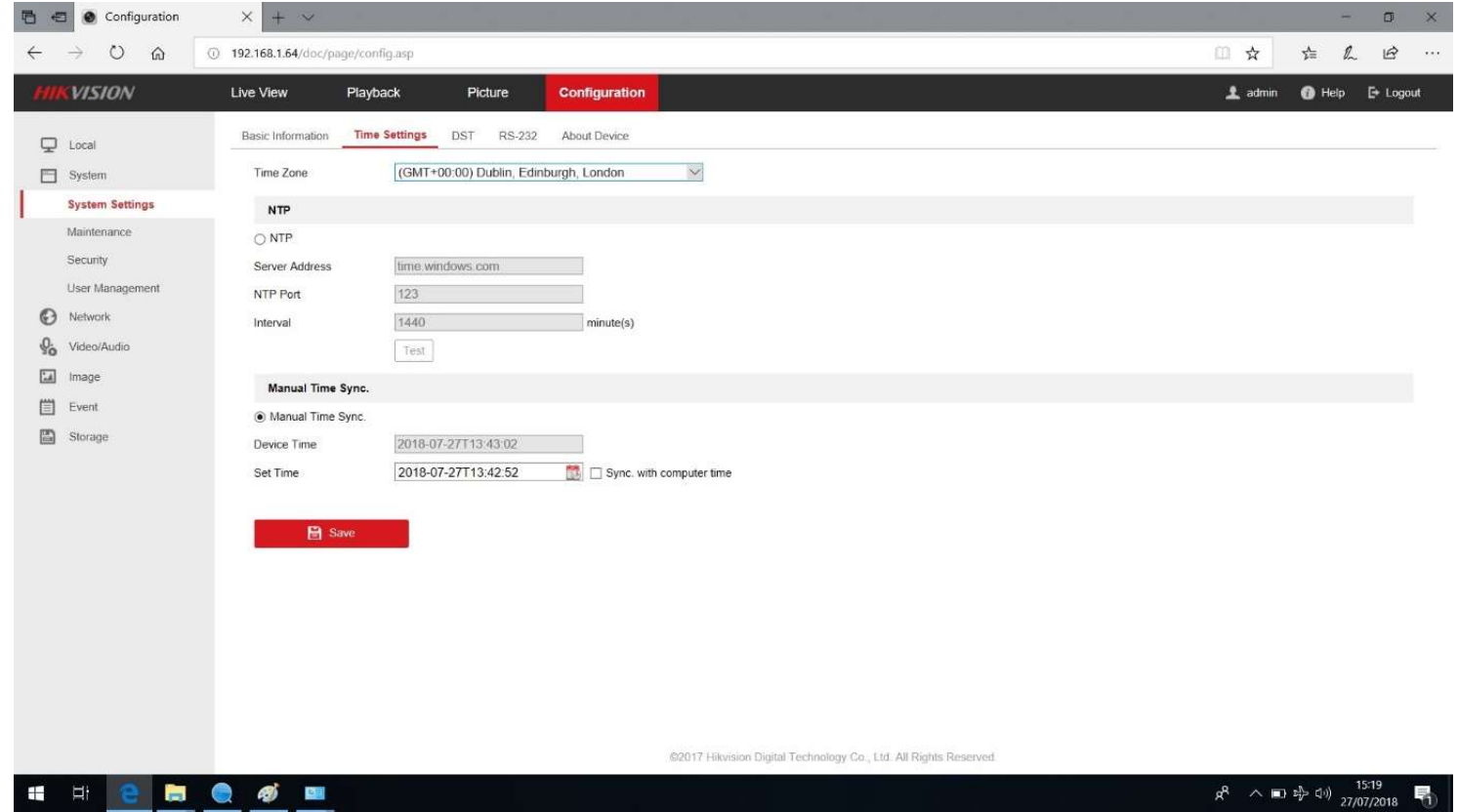

15. Now modify the Video/Audio settings. Change resolution to $1920 \times 1080 \mathrm{P}$ and Click Save. 


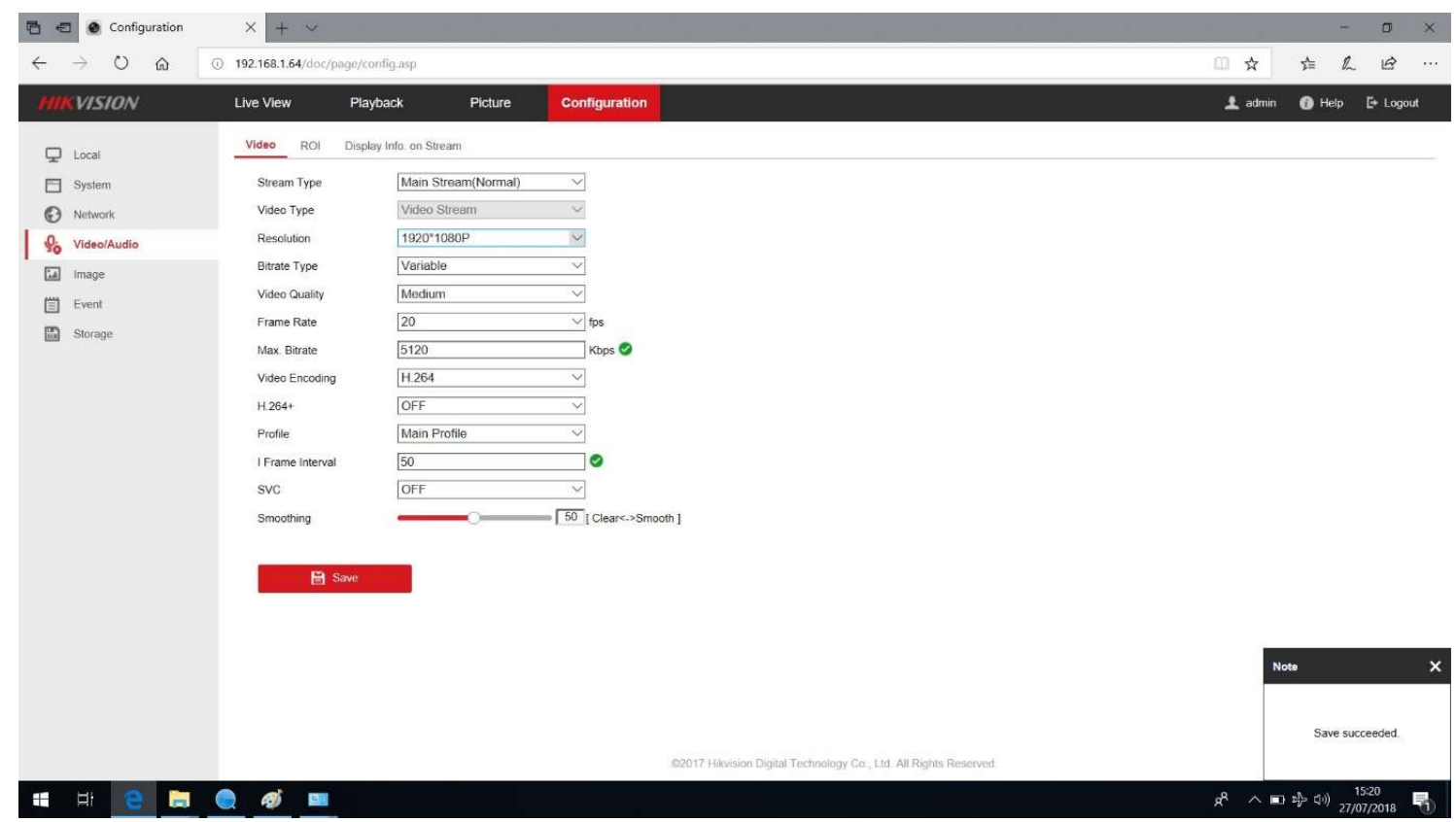

16. Go to Image / OSD settings: Change the Camera Name to something meaningful (Download and install the plug-in if necessary. If after install you can't see the image, click on the 3 dots on the right side of Microsoft Edge window and select 'Open with Internet Explorer' and allow access to the .exe). This will be displayed on the video feed. Alternatively untick the 'Display Name' box. When changes are completed Click Save.

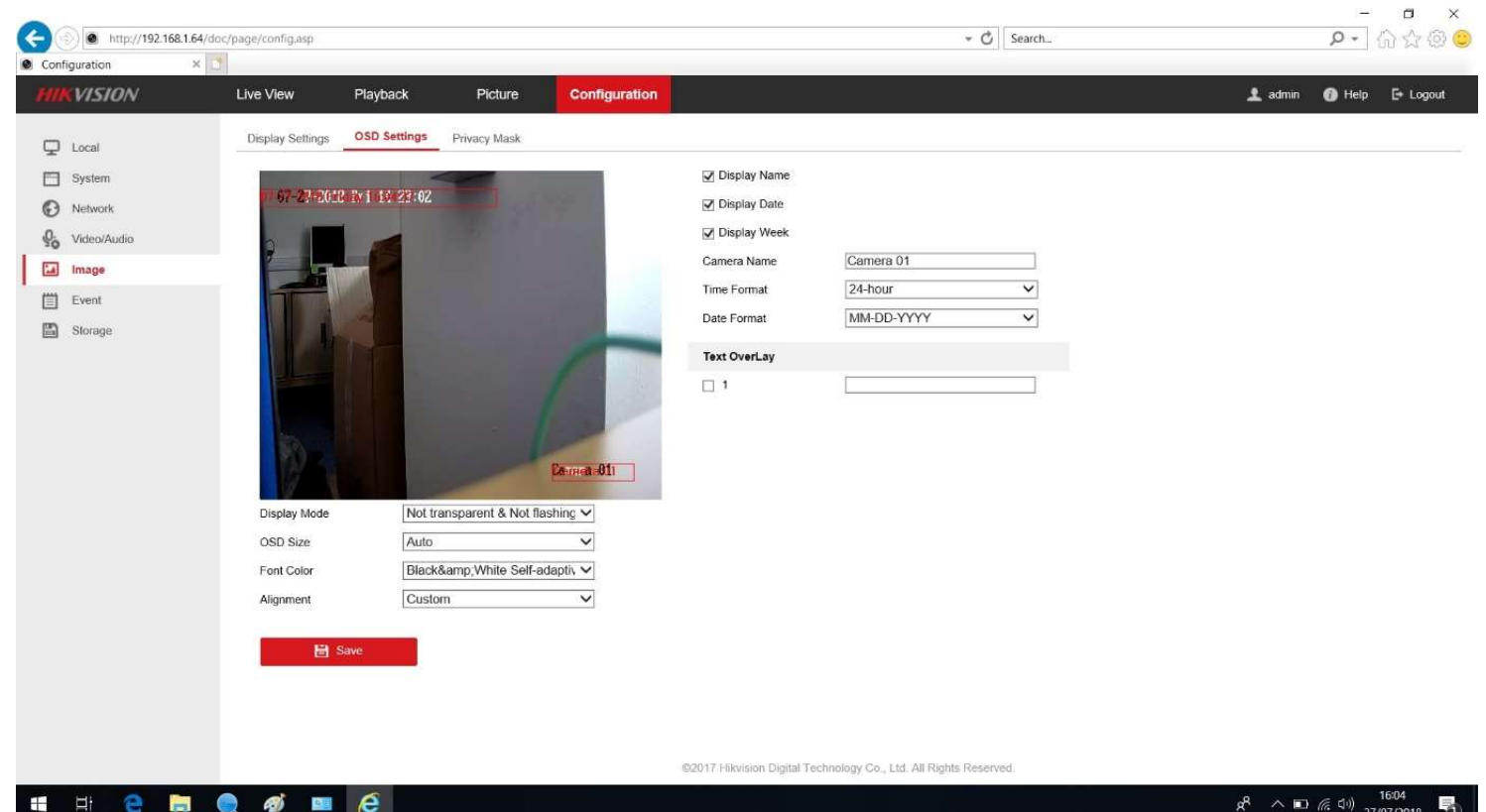

NOTE: Sometimes when we click modify a 'time-out- dialogue box will appear. In this case click 'Modify' again. The parameters modification completion dialogue box should finally display.

17. Now we modify the network configuration. 
18. In basic settings, change the subnet mask, and gateway to match those that are assigned to eth0 on the $\mathrm{Pi}$. For the test camera these were assigned as: 255.255.0.0; 169.254.217.1. Also change the IP address to 169.254.217.100. Ensure these settings are saved.

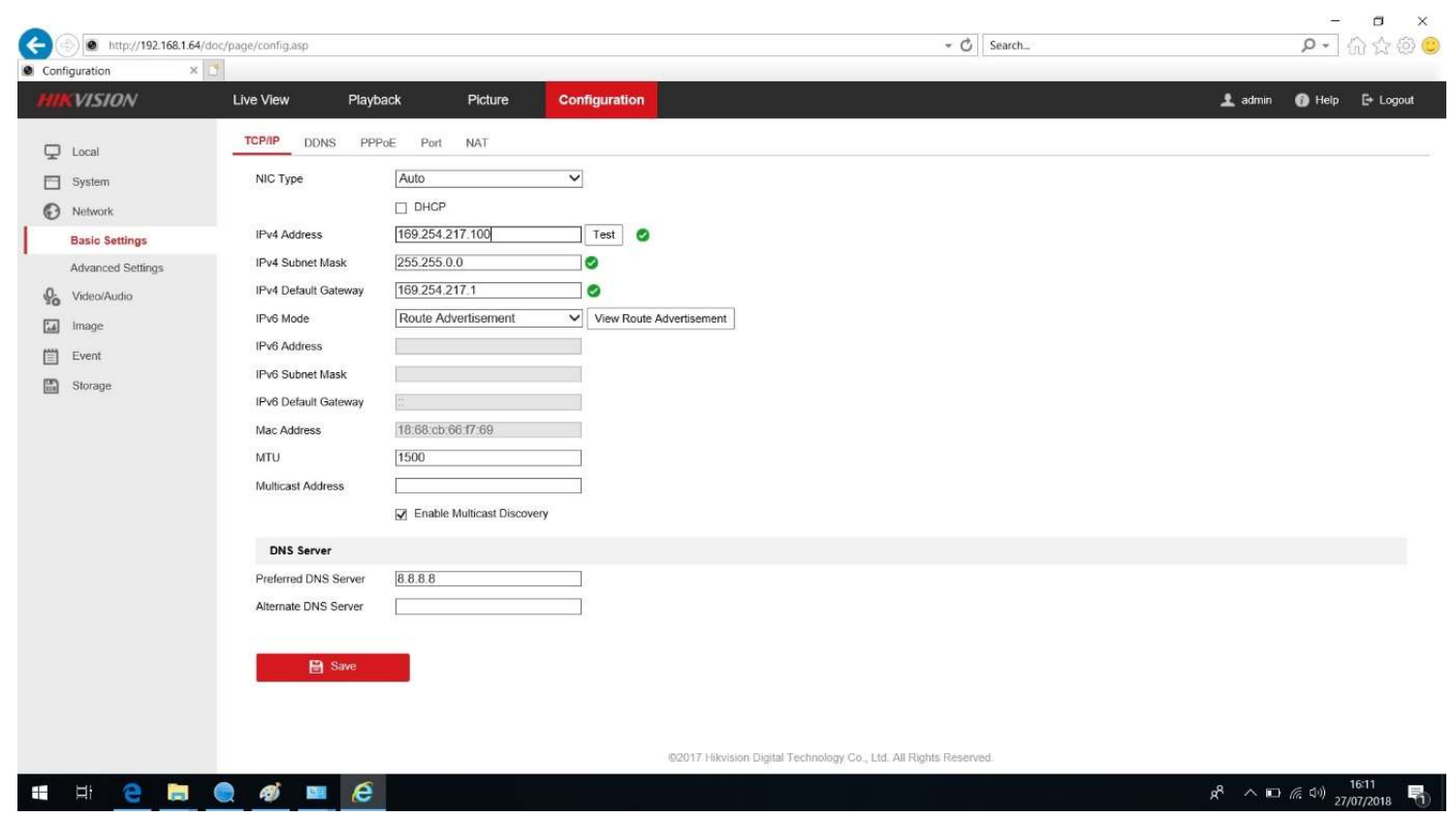

19. Close the SADP webpage and software. Change all network connection settings to their default and re-establish your internet connections (if previously available). 


\section{General assembly procedure}

1. Place the pre-installed SD card into the Raspberry Pi.

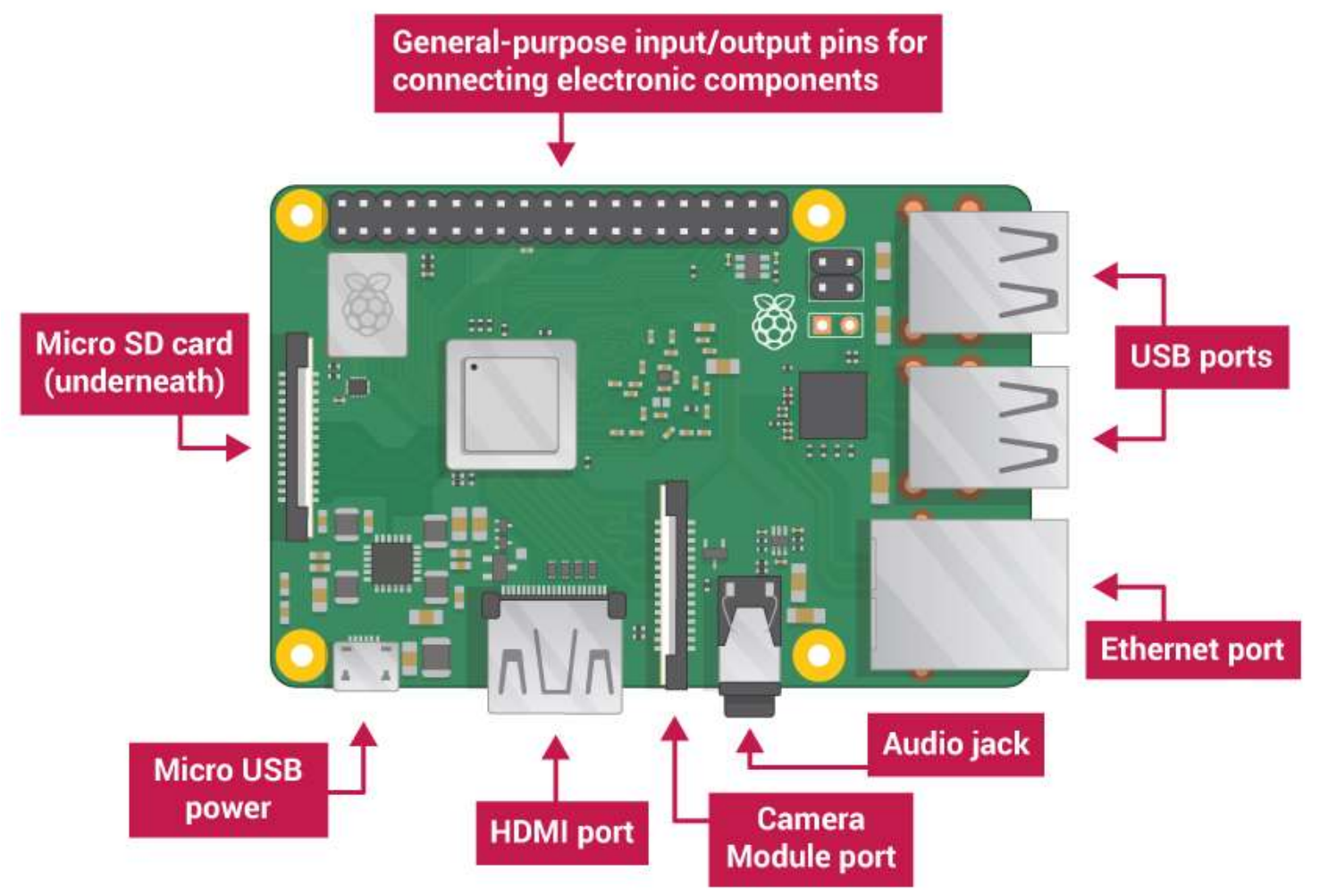

2. Mount the connection board on the Raspberry Pi

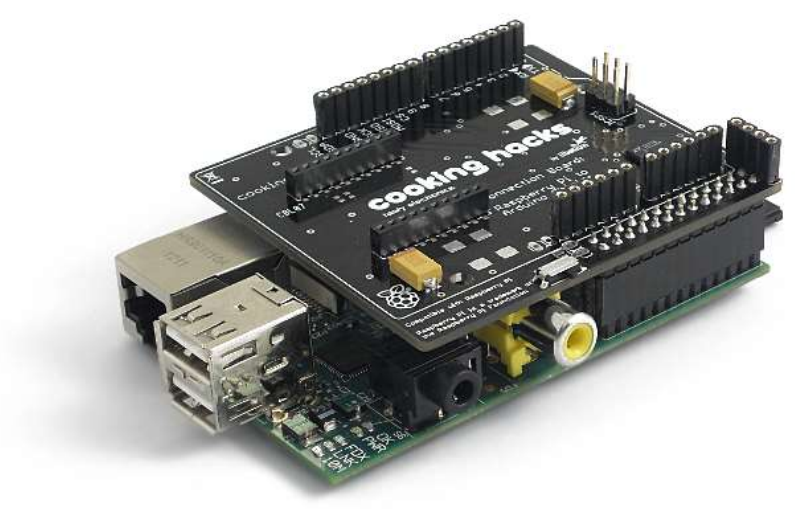


3. Assemble the pieces of the modem (antenna, SIM card, 3G/GPRS modem) and connect it to Raspberry Pi using micro USB - USB cable.
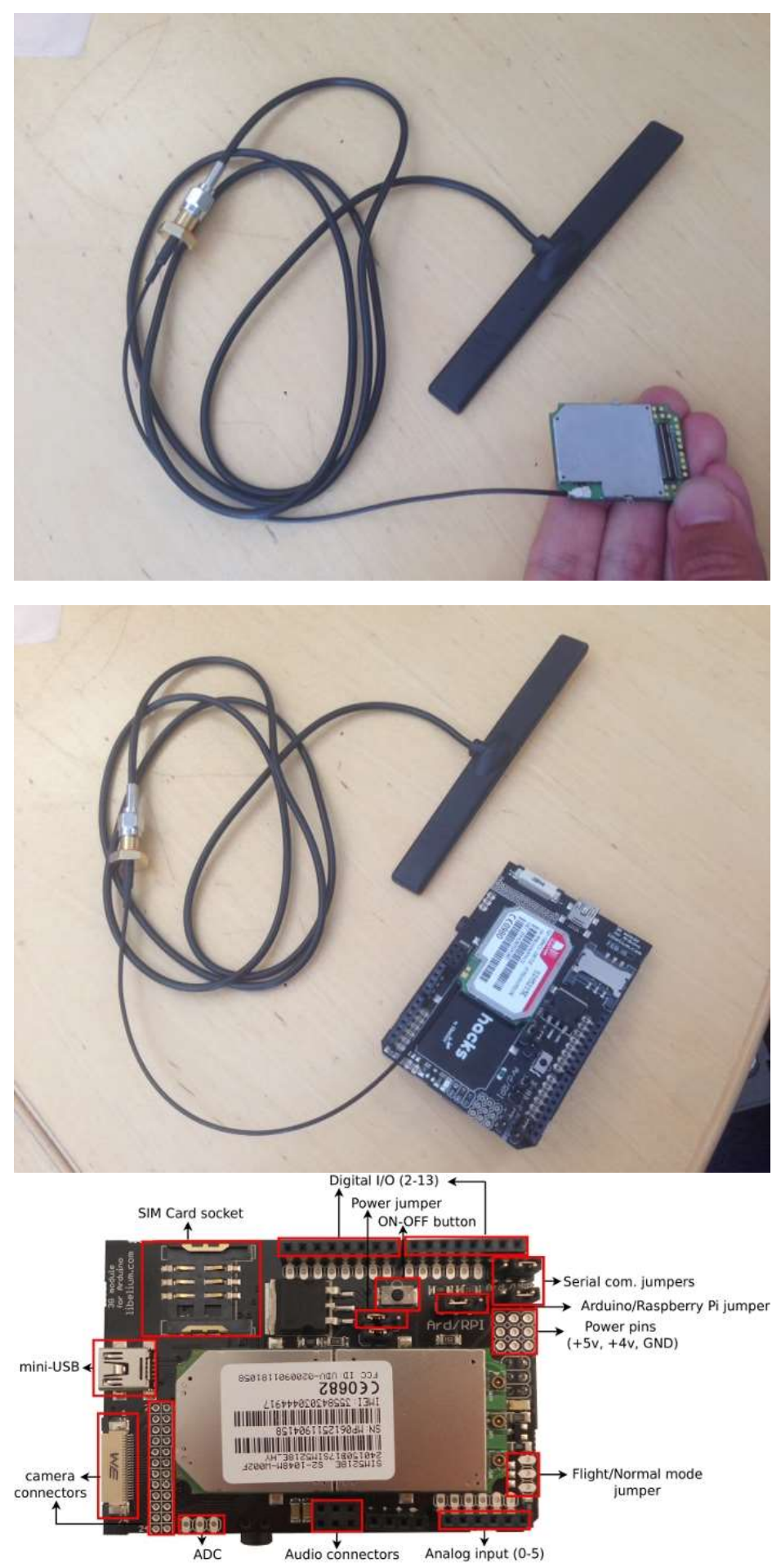
4. Connect the converter to the Raspberry Pi with the micro USB port.

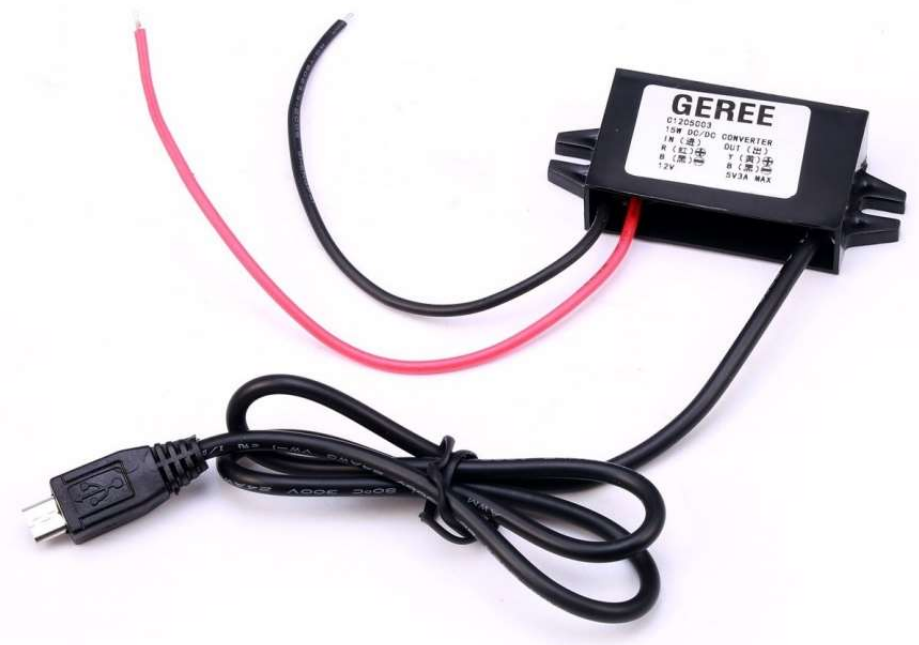

5. Prepare the following wiring to power the Raspberry Pi and the ultrasonic sensor with $5 \mathrm{~V}$ instead of $12 \mathrm{~V}$. 


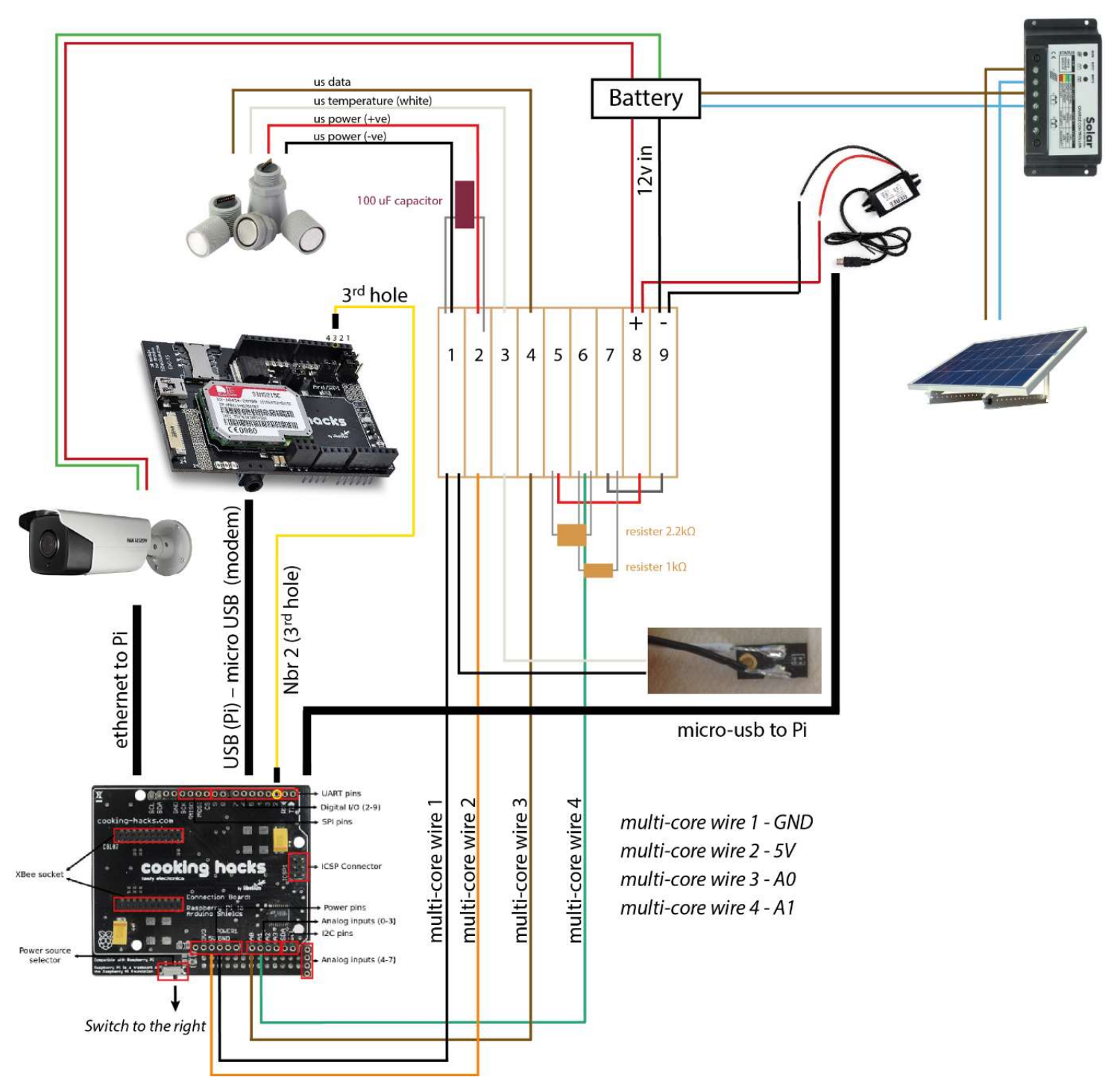

$\triangle$ Please note that the images slightly differ from the wiring diagram, follow the wiring diagram.

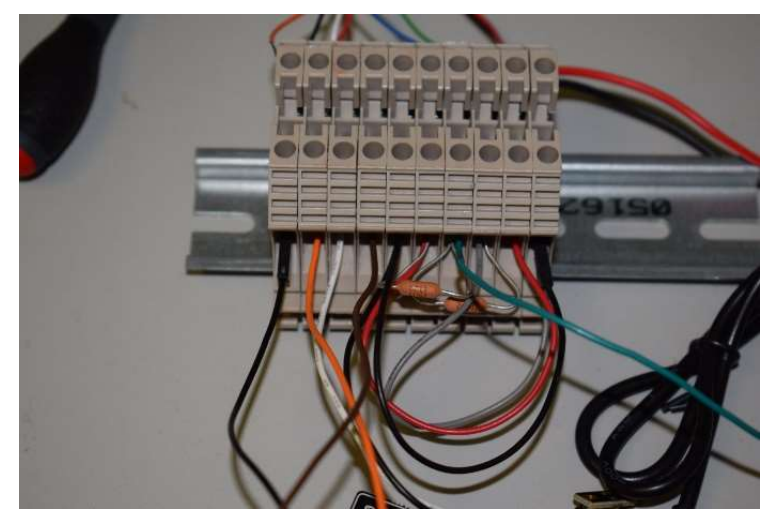

The white and black cables are connected to the temperature sensor. These two cables have to be soldiered on the sensor previously. 

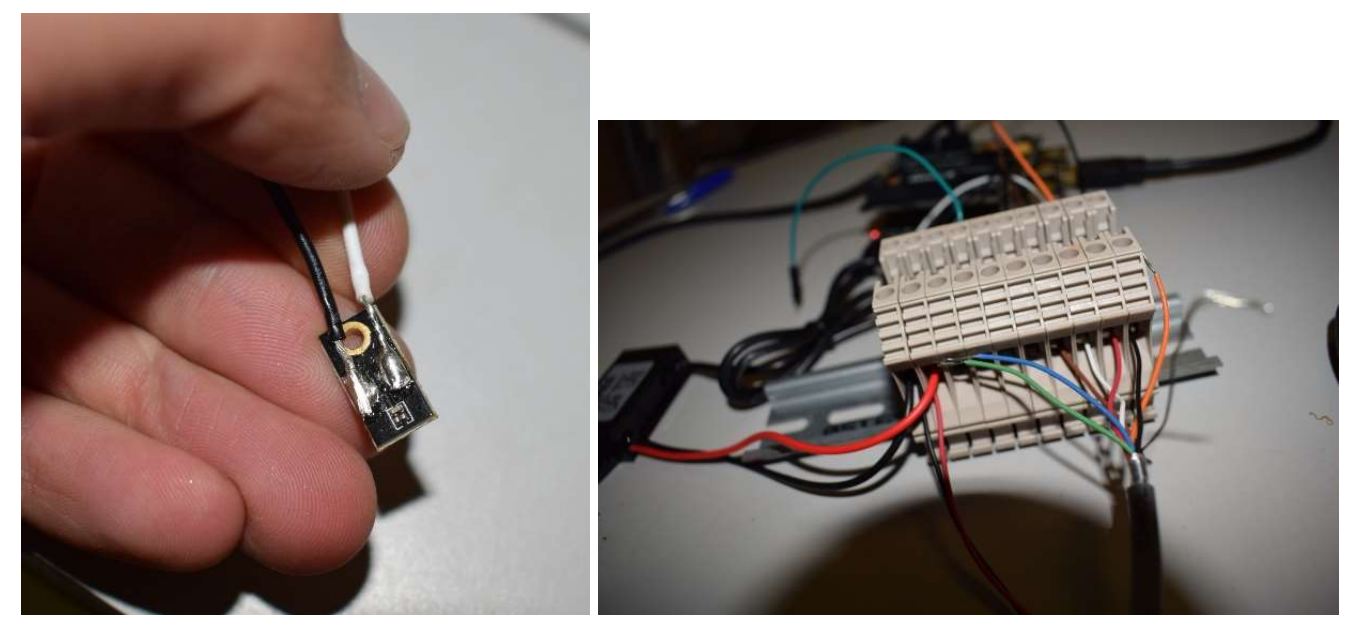

Add a capacitor to the red and black cable of the ultrasonic sensor. The positive (longer) part goes to the red cable, the negative (short) one to the black.

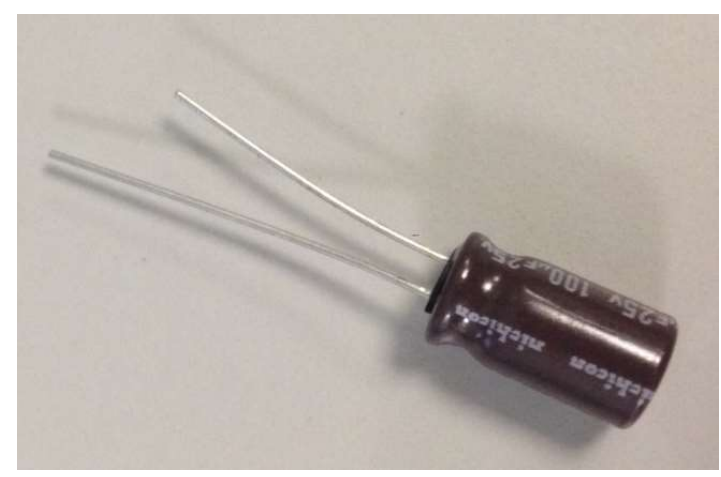

Finally connect the 3 cables of the ultrasonic sensor (brown, orange, black) and the green (measuring the charging level of the battery) to the connection board (see wiring diagram). 

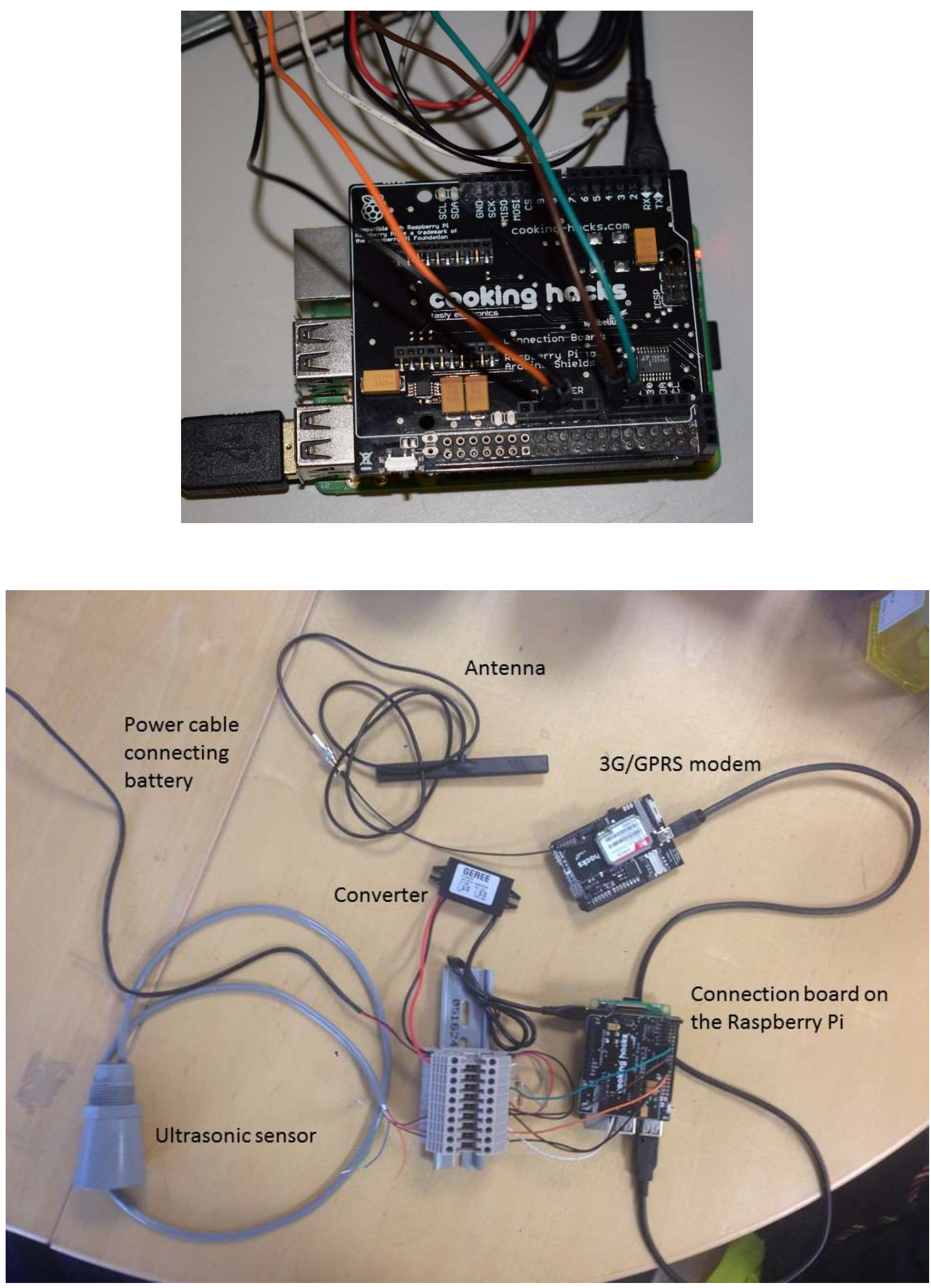

6. The camera is powered directly from the battery. Connect the camera to the battery and use the Ethernet cable to connect the camera to the Raspberry Pi. 
7. Join the solar controller to the solar panel, and then relate the controller to the battery. $\triangle$ Be careful on a sunny day, the cable is live!

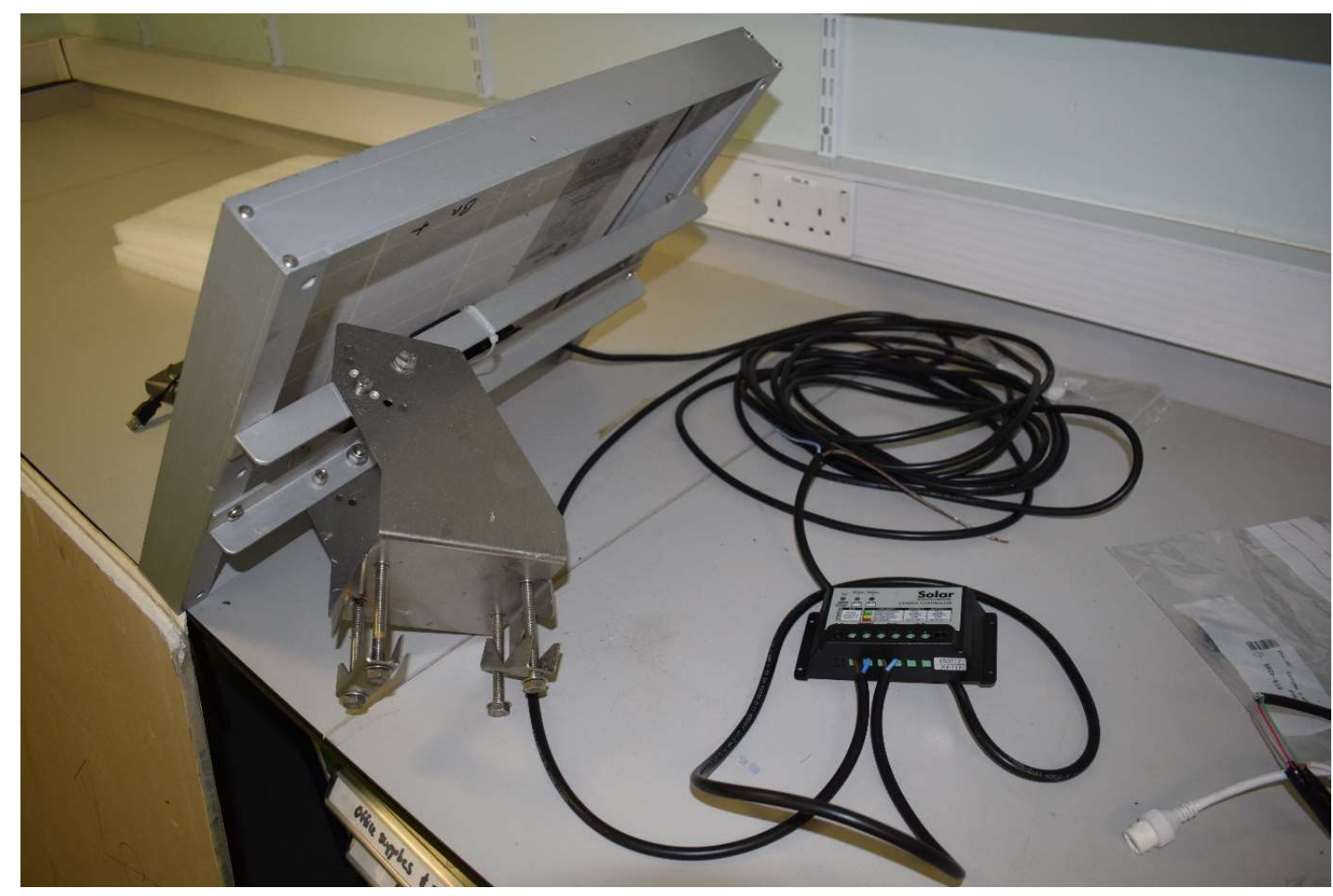

8. The solar panel cabling is prepared in the following way.

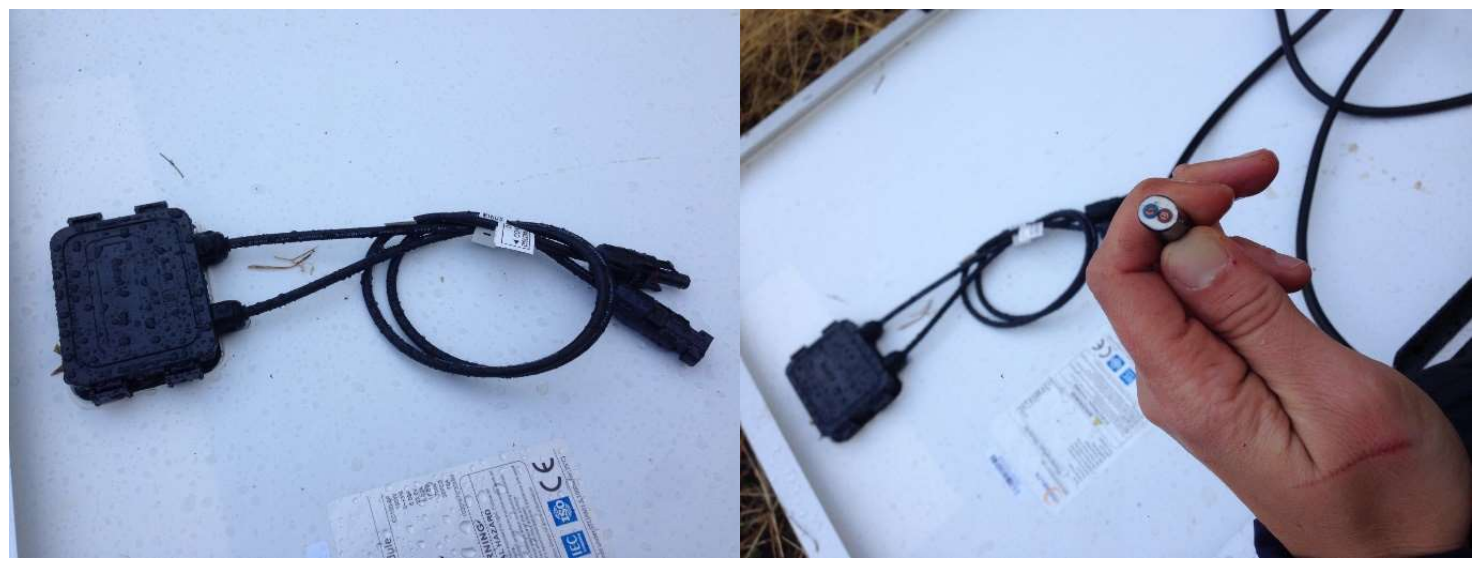



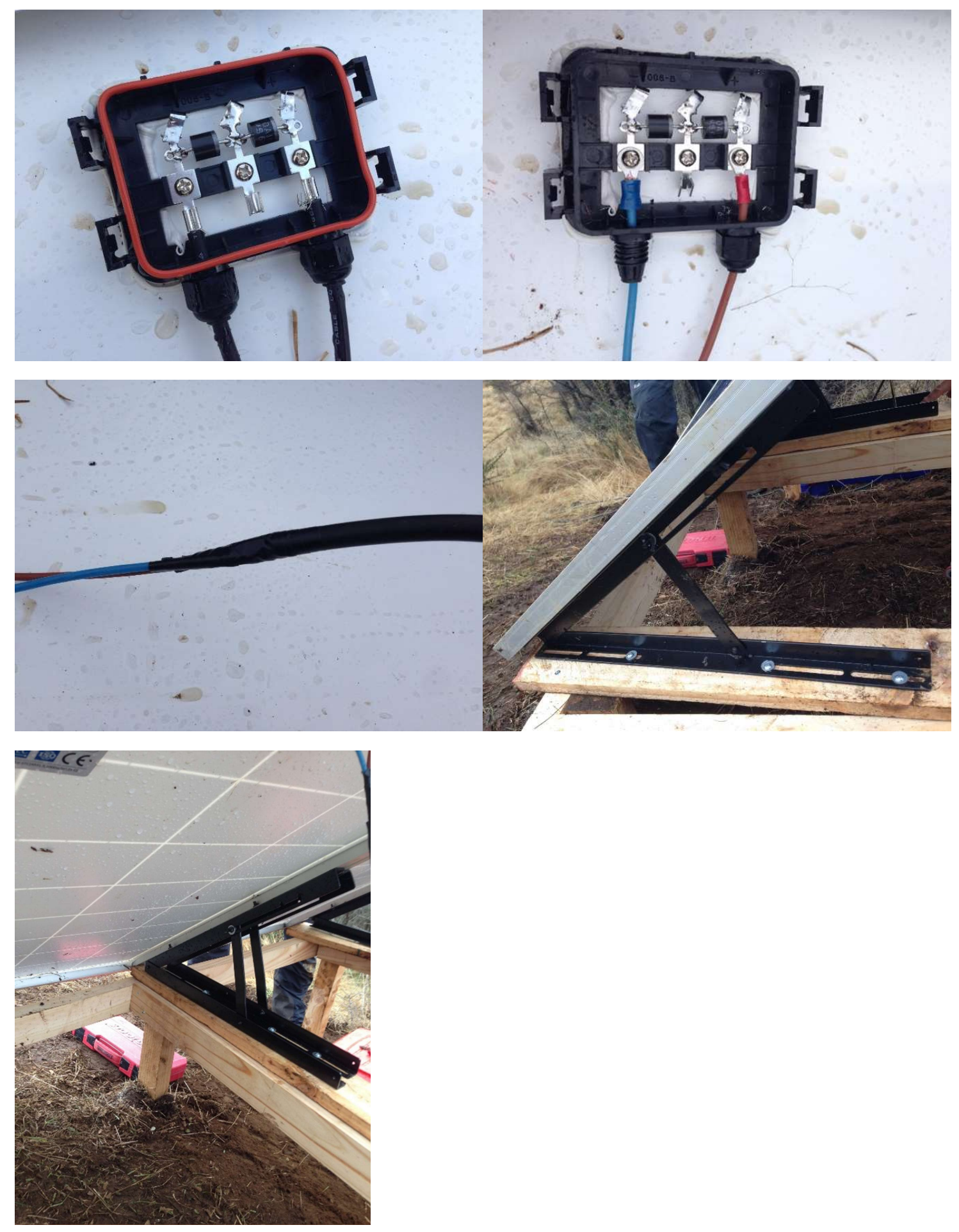


\section{Field installation}

1. Set up the system as described earlier

2. Place the SD card in the Pi

3. Prepare the Ethernet cable the following way and connect the system to the power

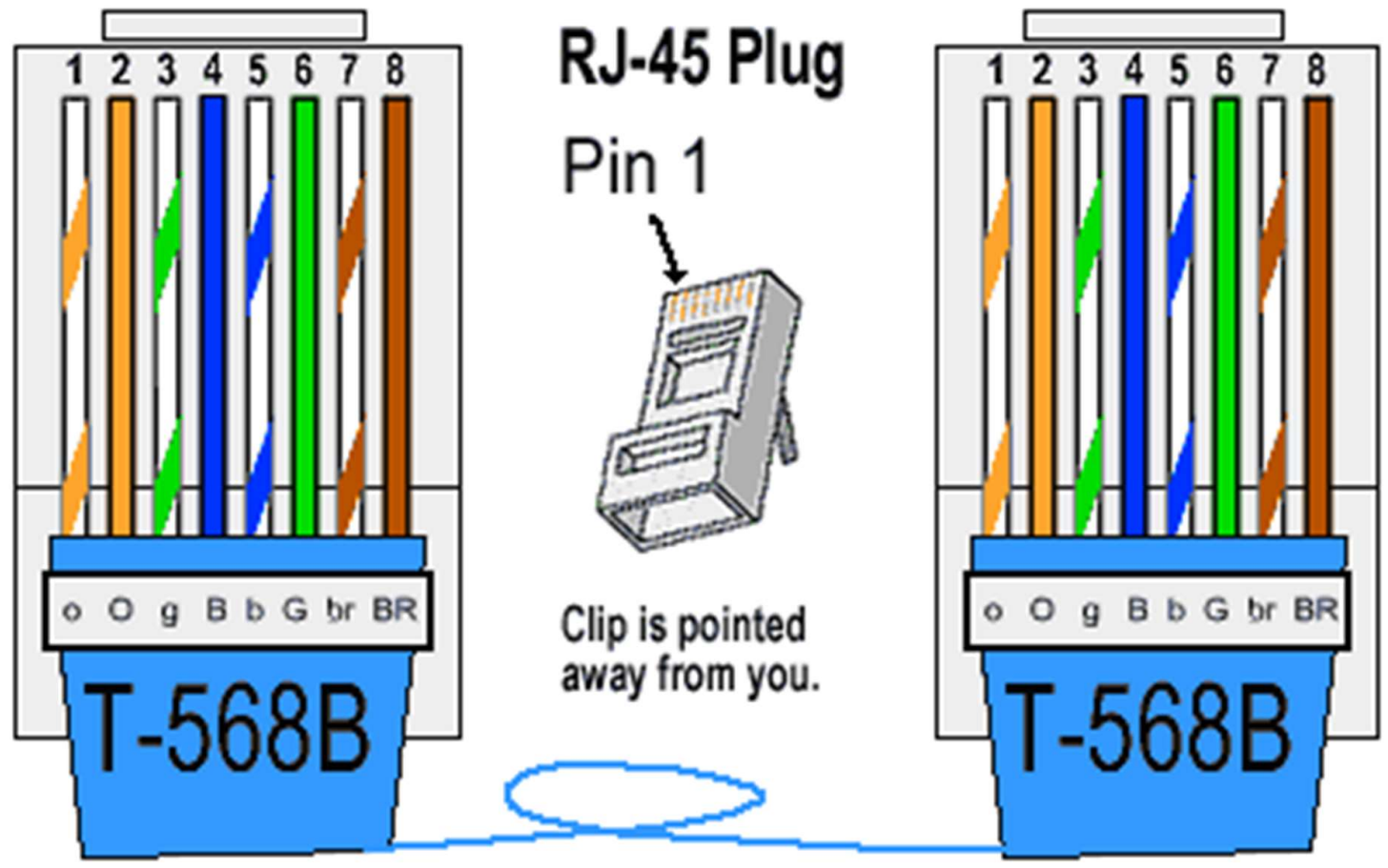

\section{Site selection criteria and advice for install}

- Ideally choose a site where you can detect the flow moving with your eyes. It is helpful if some floating features (e.g. white foam, standing waves, plant material) are present on the water surface.

- Check that you can see all the river or channel surface.

- Check mobile phone reception at the site.

- Favour installation where main power can be used instead of solar panels. It will be more reliable and will need less maintenance.

- Place the camera in an elevated position.

- The camera needs a clear view on the river; avoid for example branches within camera view and position the camera so it is not too far from the river to reduce the impacts of weather conditions which may lower visibility.

- If possible, set-up the camera in a way that the entire width of the channel is visible.

- Avoid facing the camera to the sun, reflection might cause an issue. 
- Consideration is needed for solar panel installation which should be located in a secure location well above inundation level but as close to the camera as possible in order to minimise the power requirements which increase with longer cables.

- The distance between the camera and the grey box should not exceed $20 \mathrm{~m}$, the ultrasonic sensor $300 \mathrm{~m}$.

\section{Access to camera from distance}

You can connect to your camera to view a real-time image using iVMS-4500 app by using the IP address, the port and the password of the camera.

\section{Acknowledgements}

This handbook was funded by UK Natural Environment Research Council grant $\mathrm{NE} / \mathrm{R009481/1}$ 'Real-time low-cost monitoring for hydro-geomorphological risk reduction in Chile'.

\section{$10 \underline{\text { References }}$}

Le Coz, J., Hauet, A., Pierrefeu, G., Dramais, G., and Camenen, B. 2010. Performance of Image-Based Velocimetry (LSPIV Applied To Flash-Flood Discharge Measurements In Mediterranean Rivers, Journal of Hydrology, 394, 42-52.

Major, J.J. Bertin, D., Pierson, T.C., Amigo, A., Iroume, A., Ulloa, H., Castro, J. 2016. Extraordinary sediment delivery and rapid geomorphic responsefollowing the 2008-2009 eruption of Chaiten Volcano, Chile. Water Resources Research. 10.1002/2015WR018250.

Muste, M., Fujita, I., and Hauet, A. 2008. Large-scale particle image velocimetry for measurements in riverine environments. Water Resources Research, 44 (4), W00D19.

Perks, M.T., Russell, A.J. and Large, A.R.G. 2016. Technical Note: Advances in Flash Flood Monitoring Using UAVs. HESS Discussions. 1195541121 Petit-Breuilh, M., 1999. Cronología Eruptiva Histórica de los volcanes Osorno y Calbuco, Sernageomin Boletín, 53: 46.

Tauro, F. and Grimaldi, S. 2017. Ice dices for monitoring stream surface velocity. Journal of Hydro-environment Research, 14, 143-149.

UNISDR. 2015. United Nations Office for Disaster Risk Reduction annual report.

Wilcox A.C., Escauriaza C., Agredano R., Mignot, E., Zuazo, V., Otárola, S., Castro, L., Gironás, J., Cienfuegos, R. and Mao, L. 2016. An integrated analysis of the March 2015 Atacama floods. Geophysical Research letters. 